\title{
Ariel planetary interiors White Paper
}

\section{Ravit Helled $^{1}$ (D) . Stephanie Werner ${ }^{2}$. Caroline Dorn ${ }^{1}$ (D) . Tristan Guillot ${ }^{3}$ (D) . Masahiro lkoma ${ }^{4}$ (D) . Yuichi Ito ${ }^{5}$ (D) . Mihkel Kama ${ }^{6}$. Tim Lichtenberg ${ }^{7}$ (D) . Yamila Miguel $^{8,9}$ (D) . Oliver Shorttle ${ }^{10}$. Paul J. Tackley ${ }^{11}$. Diana Valencia ${ }^{12}$ (D) . Allona Vazan ${ }^{13}$ (D)}

Received: 30 June 2020 / Accepted: 16 March 2021 / Published online: 21 May 2021

(C) The Author(s) 2021

\begin{abstract}
The recently adopted Ariel ESA mission will measure the atmospheric composition of a large number of exoplanets. This information will then be used to better constrain planetary bulk compositions. While the connection between the composition of a planetary atmosphere and the bulk interior is still being investigated, the combination of the atmospheric composition with the measured mass and radius of exoplanets will push the field of exoplanet characterisation to the next level, and provide new insights of the nature of planets in our galaxy. In this white paper, we outline the ongoing activities of the interior working group of the Ariel mission, and list the desirable theoretical developments as well as the challenges in linking planetary atmospheres, bulk composition and interior structure.
\end{abstract}

Keywords Ariel · Planetary interiors · Planet composition · Atmosphere-interior interaction

\section{Introduction}

The Atmospheric Remote-sensing Infrared Exoplanet Large-survey (Ariel) mission will measure the atmospheric composition of a large number of exoplanets with different masses and radii (e.g., [25, 143]). A key question to be addressed by the Ariel mission is 'What are planets made of?'

Understanding the connection between the bulk composition and atmospheric composition of planets and how they are linked to the planetary origin is a key topic in planetary and exoplanetary science. Determining the atmospheric composition of planets is critical for constraining the planetary bulk composition, which can then be

Ravit Helled

rhelled@physik.uzh.ch

Extended author information available on the last page of the article. 
linked to its formation process and planetary evolution (e.g., [148]). Determining the atmospheric composition of many exoplanets will provide an additional constraint to structure models, and can be used to put limits on elemental abundances in the deep interiors of planets. While it is not possible to uniquely determine planetary composition and internal structure from remote measurements, the atmospheric composition adds another piece of information and can break some of the degeneracy. In this regard, a clear strength of the Ariel mission is that it will provide statistics. A large enough sample of planetary atmospheres can be used to better understand the trends, in particular, how the planetary composition depends on the planetary mass, the orbital period, and the stellar properties such as stellar type, metallicity, and age.

The three aspects of formation, evolution and internal structure are interconnected. The formation environment and epoch determine the total availability of each chemical element. The formation process determines the primordial internal structure and thermal state of the planet. This determines the heat transport mechanism as well as the potential re-distribution of the materials, and the planetary long-term evolution (contraction and cooling rate). The planetary evolution then determines current-state internal structure. In particular, the interaction between the atmosphere and the deep interior. The long-term evolution could be responsible for the formation of secondary atmospheres as well as for atmospheric loss. Therefore, in order to link the planetary internal structure and to understand the connection between the atmosphere and the interior today, a good understanding of the planetary origin and evolution is required.

From a planetary composition perspective the questions that will be addressed with Ariel include:

- What can the M-R relation together with atmospheric measurements tell us about the planet's bulk composition?

- Under what conditions does the atmospheric composition represent the composition of the deep interior?

- Is atmospheric composition able to distinguish planetary archetypes, such as mini-Neptunes versus super-Earths?

- How can we use the knowledge of exoplanets to better understand our own planetary system and vice versa?

- How do we extract the similarities/differences between solar system (terrestrial) planets and exoplanets from atmospheric element abundances?

- How do planetary atmospheres in hot conditions evolve?

Not only is connecting planetary atmospheric compositions with bulk compositions challenging, but there will also be clear differences between various planetary types (mass, orbital period, etc.), and as discussed above, with a planet's formation history, and subsequent evolution and internal structure. Key questions to be answered by Ariel with the suggested targets are summarised in Table 1. The colours indicate whether the question addresses composition (red), evolution (blue) or origin (green), or a combination of these aspects. The first three questions are fundamental: "What are exoplanets made of?" "How do exoplanets form?" and "How do exoplanets evolve?" These questions are expected to remain open for a few decades, and even with Ariel and other future missions, unique and clear answers might not 


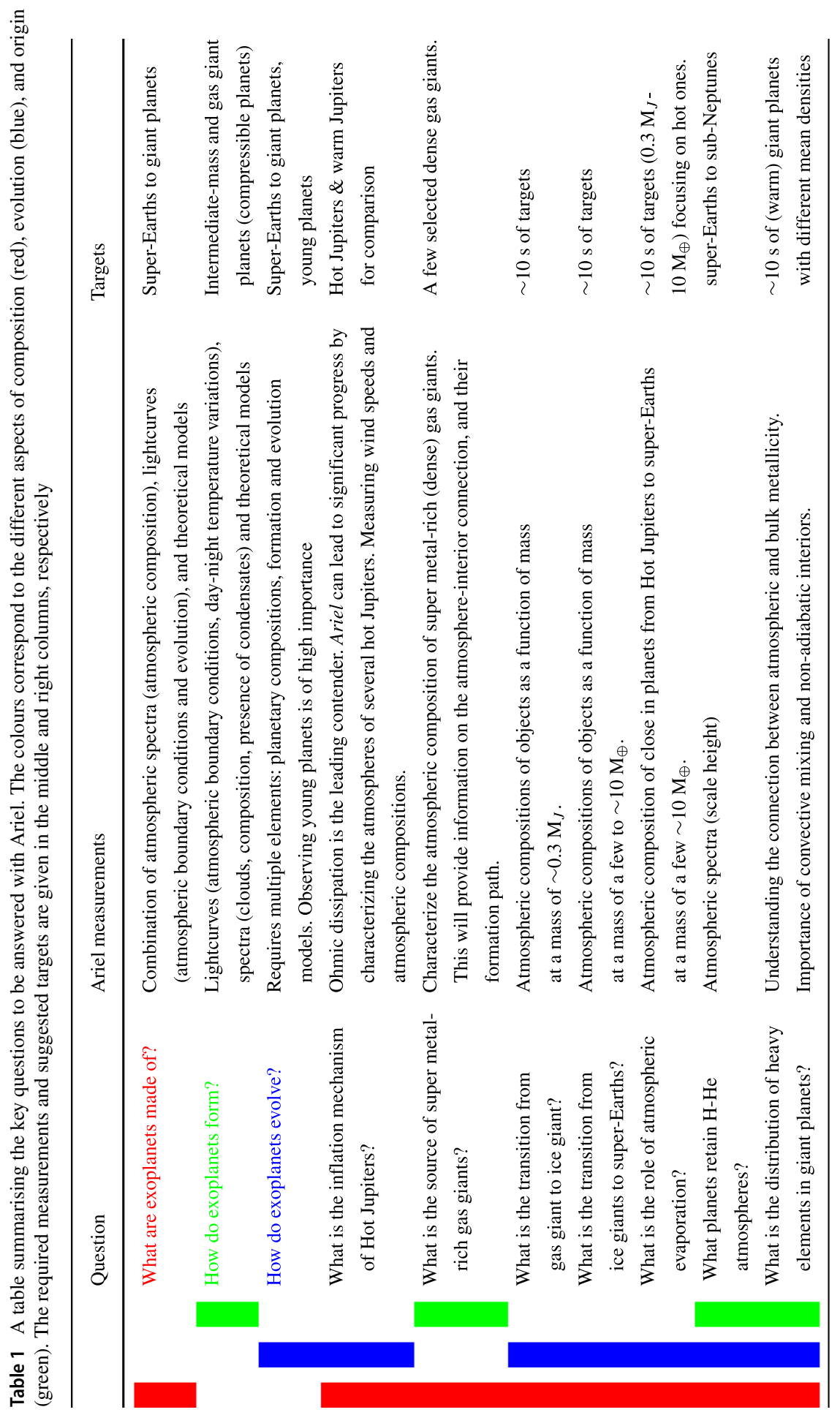




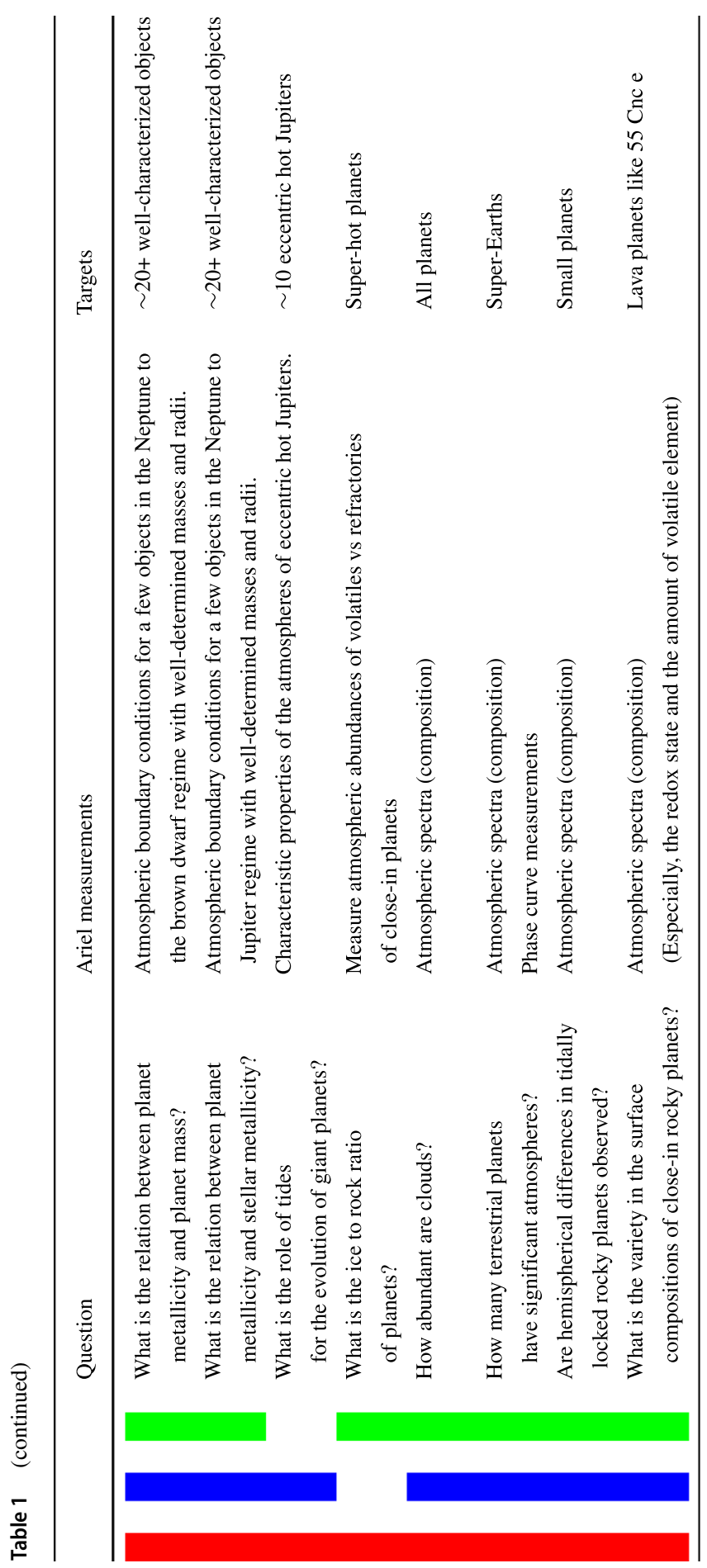


be available. However, more specific questions, as listed in Table 1, can slowly be answered providing a more complete understanding of planets and the connection between composition, formation and evolution. Since the three aspects are linked, often answering a question reflects on the three of them. As a result, many of the questions listed in the table involve more than one colour representing the three fundamental questions.

Figure 1 shows the mass-radius (M-R) relation of planets up to a mass of 120 $\mathrm{M}_{\oplus}$ as presented by Otegi et al. [111]. Identified are the "terrestrial planets" whose compositions are dominated by refractory materials, the gaseous (giant) planets that are mostly composed of hydrogen and helium (H-He), and an intermediate population (transitional planets) that includes planets that are massive Earth-like planets (superEarths) and Neptune-like planets as well as smaller version of the ice giants (miniNeptunes).

Below we discuss the research conducted related to the questions relevant for Ariel science in terms of interiors. We organize the discussion based on the masses of the objects as shown in Fig. 1: (i) Giant planets are the planets for which we currently

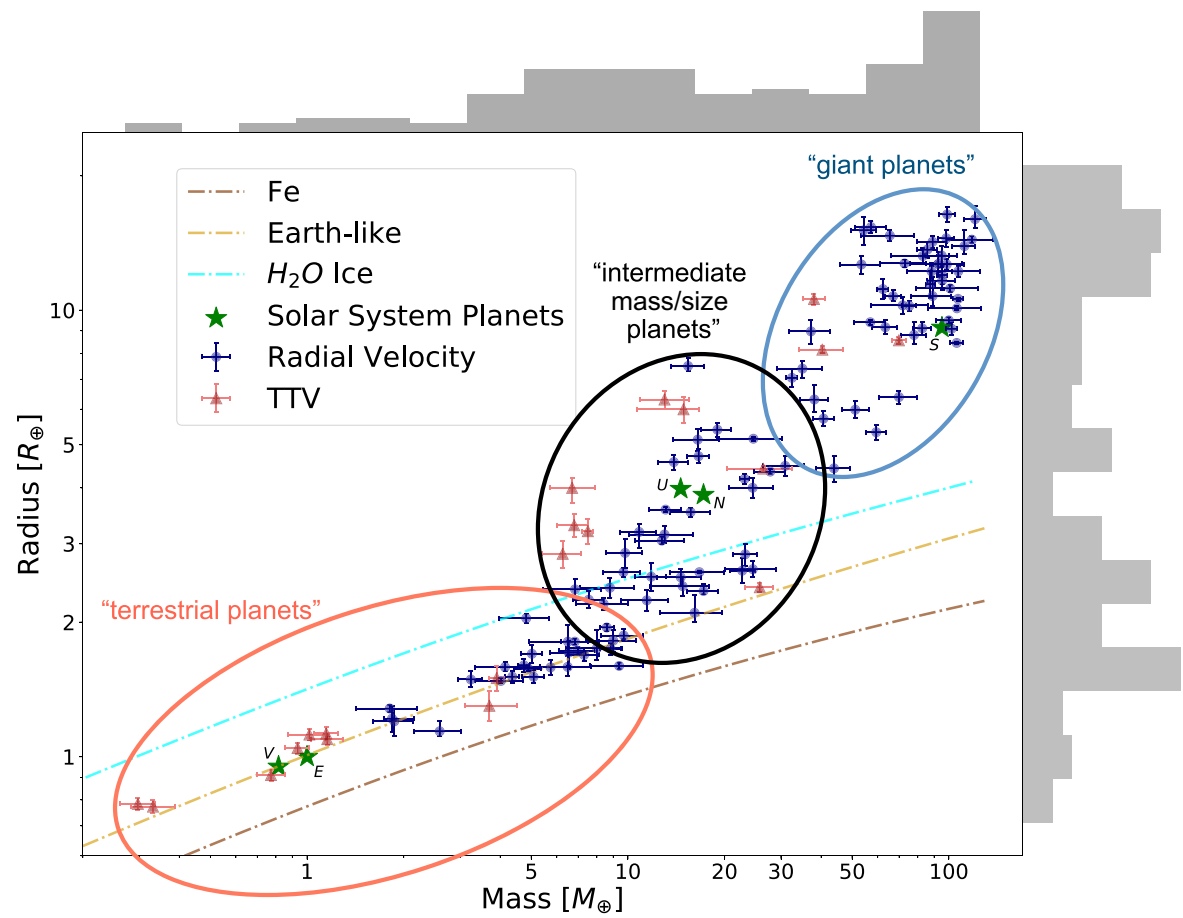

Fig. 1 The M-R diagram of planets with robust mass measurements with relative uncertainties smaller than $25 \%$ for mass and smaller than $8 \%$ for radius. The red triangles and blue circles correspond to data with mass determination from TTVs and RVs, respectively. Also shown are composition lines of pure-iron (brown), Earth-like planets (light-brown) and water ice (blue), and the distribution of exoplanet mass (top) and radius (right). We indicate the planets that are expected to be "terrestrial" "gaseous" or "intermediate" in terms of composition (from [111]) 
have the most data. (ii) Intermediate-mass planets have slightly smaller radii and are thus not as well characterized, but their high occurrence rate in the galaxy [37] should ensure a large number of potential targets to study with Ariel. (iii) Super-Earths represent the most challenging class of objects for Ariel, but they are a milestone towards the characterization of planets more similar to our own Earth.

Our paper is organised as follows. Progress related to gas giant science is discussed in Section 2. Intermediate-mass planets are presented in Section 3. Science related to terrestrial planets is discussed in Section 4. Ariel is discussed in Sections 2, 3 and 4. Finally, a summary is presented in Section 5.

\section{Giant planets}

When it comes to gas giant planets whose compositions are dominated by hydrogen and helium (hereafter, H-He), the planetary bulk metallicity is typically characterised by the metallicity, i.e., the mass fraction of heavy elements within the planet. Giant planets are key to understand the formation of planetary system: Dynamically, their migration shaped the final planetary systems, be it our Solar System (e.g. [147]) or exoplanetary systems (e.g. [85]). The gaseous envelopes of giant planets were accreted during the first millions of years of the formation of planetary systems so that the study of their bulk and atmospheric composition informs us on the conditions that led to the formation of planetary systems (e.g. [42]).

Because giant planets are $\mathrm{H}-\mathrm{He}$ dominated and are compressible they contract as their interior progressively cools (e.g., [35, 44, 60, 156]). This implies that determining their bulk composition from a measurement of mass and radius also requires knowledge of age, equations of state, and atmospheric boundary conditions (e.g., [41]). Ariel's observations are thus crucial in the sense that they complement precise determinations of radii of transiting planets and ages of their parent stars, in particular as expected from the Plato mission [117].

\subsection{Importance of the atmosphere}

The atmosphere is the external boundary condition used by interior models to calculate the overall structure of a giant planet. It is also a lid that governs how interior heat is progressively radiated away. A proper characterization of the atmosphere is therefore crucial to infer the properties of the planet's interior and its formation. For interior models, we seek to obtain with Ariel several key properties of the atmospheres of giant planets:

- Their albedo, which is the main factor governing the equilibrium temperature and therefore atmospheric entropy (e.g., [44]).

- Their day-night and equator-to-pole temperature contrasts, which also govern the rate at which interior heat can leak through (e.g., [43, 119]).

- Cloud content and wind properties since these also affect how irradiation energy is absorbed and redistributed in the atmosphere (e.g., [4, 6, 118, 134, 169]). 
- Generally, any variation in atmospheric properties, in particular abundances, tracing both the radiative and dynamical properties of the atmosphere (e.g., [26, 113]).

\subsection{Inflation of hot-Jupiters}

The importance of the atmosphere was highlighted by the works of Bodenheimer et al. [6] and Guillot and Showman [43] who showed that the radius of the famous hot Jupiter HD 209458 b was larger than predicted by standard evolution models. This inflation of Hot-Jupiters is highlighted with every newly discovered highly irradiated giant planet. Figure 2 demonstrates the clear correlation between the irradiation received by the planet and the decrease in bulk density. Figure 2 also shows that planets with equilibrium temperature above $1200 \mathrm{~K}$ have a difference between the observed radius and the one that results from evolution models that increases with $R \propto T_{e q}^{1.4}[19,76,77,97,141]$.

The inflation of hot Jupiters spurred a series of explanations to explain the observations. These include hydrodynamical dissipation, where the heat gets transported to the interior of the planet through vertical winds that push down kinetic energy that is dissipated into heat $[43,134]$, heat being transported by turbulent mixing in the external radiative zone [169], or vertical advection of potential temperature by deep atmospheric circulation [123, 144]. Another possibility is Ohmic dissipation, resulting from the interaction of the zonal winds with the planets' magnetic field [4, 14, 40, 59, 114, 118, 121, 168, 169]. Finally, some studies have remained agnostic towards the physical mechanism that cause the inflation, but showed how different

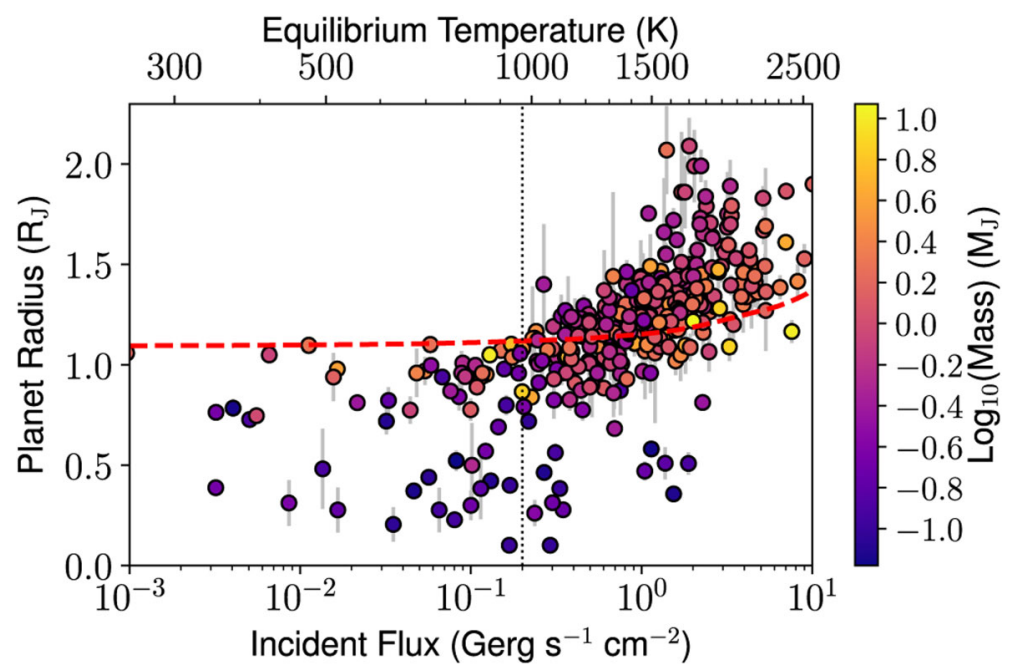

Fig. 2 Radii of transiting giant exoplanets plotted against their incident flux (or equilibrium temperature) and colored by mass on the log scale. The dashed red line is the radius of a Jupiter-mass pure H/He model with no inflation effect (from [141]) 
fractions of heat deposition in the planet's interior change its structure and observable parameters [2, 69].

A common denominator from all these studies is that the inflation of hot-Jupiters is caused by the intense stellar irradiation that these planets receive, and therefore changes in the stellar irradiation either due to stellar evolution (e.g., [39, 40, 70]) or to the planet migration - that changes their semi major axis and the received Flux(e.g., $[12,101])$, can cause a change in the inflation rate of planets, reflecting the planet history.

Ariel will aid in in this field with a better determination of composition, thermal profile and circulation in giant planet atmospheres, that will help to find correlations and identify the physical mechanisms that cause hot Jupiter inflation. This is expected to improve our understanding of the internal structure, formation, and evolution of giant exoplanets.

\subsection{Atmospheric and bulk composition}

Spectroscopic measurements of giant exoplanets allow us to further characterize these objects. These measurements, combined with accurate mass and radius determinations can be used to better understand the nature of giant planets (see e.g., [91], and references therein). The bulk composition of giant planets can be estimated from accurate measurements of the mass and radius. This estimate, however, has a relatively large (theoretical) uncertainty since it depends on the materials used to represent the heavy elements, their assumed distribution, and the equations of state used in the models as we discussed below (e.g., [3, 104, 156]). Although atmospheric measurements cannot give us the radial distribution of heavy elements directly, it can greatly illuminate this topic when compared to bulk internal enrichment inferred from the mass/radius data alone. Indeed, the presence or absence of a systematic bias between the enrichment values given by both methods would allow us to quantify the degree of compositional segregation and inform us on the strength of the mixing processes at play [152, 157].

The composition of gaseous exoplanets are typically inferred either by assuming a common mechanism for inflated hot Jupiters [13, 45] or by analysing only warm giant exoplanets, whose radii are not expected to be affected stellar irradiation [142]. However, it is not only the bulk composition that is important but the actual distribution of heavy elements within the planet. First of all, enriched envelopes have higher molecular weights and therefore shrink more effectively than when the heavy elements are concentrated in a central core. This also leads to a higher envelope opacity which leads to a less efficient cooling and slower contraction [62]. This means that at a given age, the estimated heavy-element mass from theoretical models varies depending on the model assumptions. This effect is particularly important for planets with significant enrichments and for intermediate-mass planets (sub-giants), since it can result in an overestimate of the heavy-element mass required to reproduce the measured radius $[3,156]$.

In order to take full advantage of the expected Ariel data progress in theory is required. For example, structure and evolution models should consider the uncertainties associated with the assumed opacity, assumed internal structure in particular, the 
distribution of the heavy elements, the used equation of state, and age of the planet. In addition, available opacity tables as a function of metallicity, pressure and temperature are usually sparse and thus, often internal structure models are not self-consistent in terms of the opacity calculation and the envelope metallicity. An attempt to bridge these inconsistencies was presented by [152], and a more physically-based opacity model that accounts for various compositions, grain properties and the existence of clouds for gaseous planet is still missing.

Finally, the connection between the atmospheric and bulk composition of giant exoplanets needs to be better understood. Even for the solar system gas giants, this relation is still poorly understood and being intensively investigated (e.g. [47], and references therein).

\subsubsection{Compositions of hot Jupiters}

Current exoplanet data have taught us that there is a large spread in the predicted metallicities of gas giant planets [142]. Therefore there is a clear need to identify the trends in terms of planetary mass-metallicity relation, bulk-compositionatmospheric-composition relation as well as the dependence of the stellar type and age. Ariel will provide critical information on these relations and will therefore allow us to better characterise exoplanets and will improve our understanding on the dependence of the planetary bulk and atmosphere composition on the stellar and orbital properties.

When the radius, mass, and age of a giant planet are measured by observation, the heavy-element content can be inferred through theoretical modelling of the planet's internal structure and gravitational contraction. A significant proportion of close-in giant planets detected so far are found to be quite enriched with heavy elements, which account for several tens of percent of the planetary total mass [45, 142]. The first example is HD $149026 \mathrm{~b}$, which is a sub-Jupiter of $\sim 110 M_{\oplus}$ with inferred metal content of $60-80 M_{\oplus}$ [125]. The discovery of such high density giant planets certainly gives support to the core accretion theory of giant planet formation in which a central core composed of heavy elements first forms and then captures the ambient nebular gas composed of $\mathrm{H}-\mathrm{He}$ in a runaway fashion $[5,100]$. The standard coreaccretion theory does not predict such high enrichments as inferred for high-density giant planets $[52,62]$.

One should keep in mind that the inferred composition depends on the material chosen to represent the heavy elements. As a result, the exact composition of giant exoplanets cannot be determined. Indeed, inferring the heavy-element masses in giant exoplanets strongly relies on theoretical modelling. It was recently shown by [103] that the inferred composition of giant exoplanets can significantly vary depending on the model assumptions. Large theoretical uncertainties include the used equation of state, the assumed distribution of the elements, and the atmospheric model. Another important property that should be considered is the planetary age. Its accurate determination can be used to narrow the uncertainty in the inferred composition. We therefore suggest that some of the Ariel giant planet targets should include planets around stars with a relatively good age measurement (within $\sim 10 \%$ ). This is expected to be possible thanks to the upcoming Plato mission [117]. We stress that, in order to 
take full advantage of Ariel data, progress in theory is required. Although ambiguity on the exact planetary composition are likely to remain, the large statistics expected from Ariel will be used to identify the trends and improve our understanding of giant planet origins.

\subsubsection{Origin of heavy elements in warm Jupiters}

The large amounts of heavy elements in warm Jupiters are expected to be accreted during or after the runaway gas accretion phase. At these later stages the accreted material does not settle all the way to the center (core) and instead it contaminates the gaseous envelopes. According to detailed investigation of the dynamics of planetesimals around a growing proto-gas giant, however, it is hard for a massive planet to capture large amounts of the surrounding planetesimals in situ (e.g., [132]).

The effect of planetary inward migration on the capture efficiency of planetesimals is shown in Fig. 3; the numerical results were obtained by $N$-body simulations for $10-\mathrm{km}$ planetesimals around a migrating Jupiter-mass planet in a protoplanetary gas disc [133]. It is confirmed that orbital migration helps the planet capture planetesimals. Especially for more than 50-100 $M_{\oplus}$ of heavy elements to be captured, a long-distance migration ( $~ 40$ AU) is needed, as shown in Fig. 3a. In addition, planetesimal capture is found to occur in relatively limited regions (see Fig. 3b); in particular, no planetesimal accretion occurs in inner warm regions due to strong aerodynamic shepherding. Thus, gas giants migrating over a long distance tend to capture cold materials.

This leads to a prediction that highly metal-rich gas giants may have not low $\mathrm{C} / \mathrm{O}$, but nearly stellar $\mathrm{C} / \mathrm{O}$ ratios. At such large semi-major axes in passive disks, both carbon and oxygen are contained in ice planetesimals (i.e., beyond the $\mathrm{CO}_{2}$ snowline) from a simple thermodynamic equilibrium consideration (e.g., [108]). Detailed calculations of disk chemistry also demonstrate that the $\mathrm{C} / \mathrm{O}$ ratio of ice is similar to
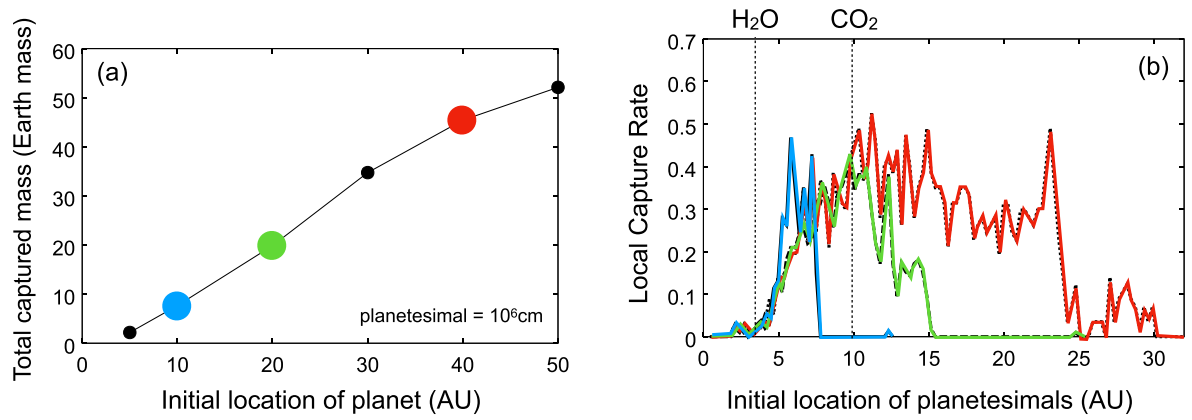

Fig. 3 Results of dynamical simulations of planetesimal capture by a migrating giant planet-The left panel shows the total mass of the planetesimals that the planet captures during its orbital migration as a function of the semi-major axis at which the planet starts migration; the right panel shows the fraction of the planetesimals located initially at an semi-major axis that the planet engulfs. The vertical dotted lines in panel (b) indicate the semi-major axes of the $\mathrm{H}_{2} \mathrm{O}$ and $\mathrm{CO}_{2}$ snowlines. Those panels have been adapted from Figure 5 of [133] 
the stellar ratio at $\gtrsim 10$ au [27]. A determination of the $\mathrm{C} / \mathrm{O}$ ratio of enriched gaseous exoplanets with Ariel can be used to test this prediction.

The radii of close-in gas giants are known to increase with decreasing distance to the central stars, on average (see e.g. [137]). This tendency is not only due to increase in stellar irradiation, but also due to unknown mechanisms for energy injection. The latter prevents us from estimating their bulk metallicities correctly. Thus, the close-in giant planets with inferred bulk metallicities are ones with moderate irradiation (socalled warm Jupiters; $\lesssim$ a few $\times 10^{8} \mathrm{erg} / \mathrm{s} / \mathrm{cm}^{2}$ in [142]). Instead of bulk composition, the atmospheric $\mathrm{H}_{2} \mathrm{O}$ abundances have been inferred for hot Jupiters with high stellar irradiation via transmission spectroscopy done with Hubble/WFC3 in the near-IR during their primary transits. Recent retrieval models for transmission spectra in the optical and near-IR of ten hot Jupiters (e.g. [115]) show that half of those atmospheres have sub-stellar $\mathrm{H}_{2} \mathrm{O}$ abundances, though the observational errors are still large. This result apparently seems inconsistent with the high bulk metallicities of warm Jupiters. However, this is still inconclusive, partly because the argument is based almost only on the water features in the near-IR; and information on other molecules such as $\mathrm{CO}, \mathrm{CO}_{2}$, and $\mathrm{CH}_{4}$ is unavailable. In addition, we do not have enough knowledge of haze and clouds, which possibly obscure the $\mathrm{H}_{2} \mathrm{O}$ features. Finally, the elements and molecules in the atmospheres of giant exoplanets are measured in the uppermost part of the atmosphere and therefore might not represent the bulk composition. Recent developments in giant planet theory suggest that giant planets are likely to be inhomogeneous and have composition gradients (e.g., [18, 47, 51, 105, 159, 163]). As a result, atmospheric composition only provides a limited glimpse into the composition of giant exoplanets.

A determination of the atmospheric $\mathrm{H}_{2} \mathrm{O}$ abundance of giant planets is important since it can be linked to their origin and evolution (e.g., [50, 93]). Collecting information on the water abundance in the atmospheres of many hot Jupiters, and comparing them to other elements (e.g., carbon) would reveal important information that could be used to constrain planet formation and evolution models. Nevertheless, connecting this information with the bulk composition remains a challenge.

\subsection{Gradual composition distribution and envelope enrichment by convective-mixing}

A main challenge in linking the atmospheric composition with the bulk is due to the fact that giant planets might not be homogeneously mixed. This possibility seems to be rather realistic for the solar-system gas giants and there is no reason to believe that giant exoplanets are significantly different. At the same time, we are still lacking an understanding of under what conditions giant planets tend to be homogeneous (mass, age, formation process). This topic should be investigated further in order to take full advantage of Ariel data.

Composition gradients in giant planets could be a result of number of physical processes such as: (1) Solids (heavy elements) accretion during the formation process (e.g. [7, 11, 16, 61, 89, 153, 154]). (2) Solubility of materials in metallic hydrogen followed by convective mixing (e.g. [135, 138, 162, 166]). (3) Helium phase separation (e.g. [34, 102, 138]). (4) Rotation and magnetic field effects [15]. 
The resulting gradual composition distribution can change with time by convective-mixing [157], which in turn leads to enrichment the outer gaseous layers with deep interior materials. The occurrence of convective-mixing depends on the ratio between the temperature gradient and the composition gradient along the interior, according to the Ledoux convection criterion [80].

Simulations of self-consistent structure and thermal evolution find convective mixing to be efficient in a large range of giant planets interiors [104, 157, 158]. However, since the mixing parameters in planetary conditions are poorly constrained, such simulations provide a range of possible solutions and the efficiency of mixing is yet to be determined. For example, mixing could take place in the form of layered-convection, which provides a lower prediction for the efficiency of mixing in giant planet interiors (e.g., $[18,79])$.

Measurements of the atmospheric abundances with Ariel for large sample of giant planets can help to constrain the parameter-space of the convective-mixing efficiency. It is known that planet current location is not necessarily its formation location. In the protoplanetary disk phase young protoplanets are expected to migrate, usually inward (e.g., [140], and many more), while they are still growing. The gas-to-dust ratio and the chemical element content of different material phases in the protoplanetary disk varies with location and time. We discuss changes with location in the disk, controlled by the temperature.

Naively, the outer envelope of a gaseous planet is composed of later accreted (current location) materials, while the deep interior composition is related to the planet's formation location. However, efficient convective mixing sweeps deep interior materials upward, and enriches the outer envelope with formation composition. Thus, the abundance of different species by the Ariel mission, will indicate on the convective mixing efficiency, and on the planet formation location.

A similar idea was used in several studies to determine Jupiter's formation location, based on its current atmospheric abundances (e.g., [107]). Modeling the evolution of Jupiter interior indeed suggests that the early accreted deep interior materials can reach the outer envelope in less than one giga year [159]. The Ariel data will allow us to perform similar studies for a large sample of exoplanets, namely to examine material abundances of chemical spices that are not expected to appear in the current planetary location. If these elements are found to be abundant the atmospheres, then it would imply that convective mixing from the deep interior is a significant process in giant planet interiors. Therefore, the Ariel mission will improve our understanding of convective mixing and material transport processes in giant planet interiors.

\subsection{Ariel observations of giant planets}

Giant planets are thus key targets to address questions linked to the composition, formation and evolution of planets identified in Table 1:

- $\quad$ The source of the inflation mechanism for hot Jupiters discussed in Section 2.2 can be better identified by fully characterising the atmospheric properties of both 
hot and warm Jupiters. Typically, several tens of targets would be needed to improve the statistics and address this topic.

- The origin of very metal-rich gas giants that require several tens $\mathbf{M}_{\oplus}$ of heavy elements is still poorly understood and reflects on their formation and evolution histories. Ariel observations of a handful of bright, super-enriched gas giants would lead to a robust determination of the planetary radius and atmospheric metallicity to decrease uncertainties in the models.

- Understanding the distribution of heavy elements in giant planets requires measuring both their bulk and atmospheric properties. A large-enough statistical sample of tens of targets with well-determined stellar and planetary properties is required.

- The role of tides in the evolution of giant planets may be addressed through the characterisation of the atmospheres of highly eccentric planets, in order to get both their atmospheric properties and atmospheric thermal evolution at periapsis. This may be achieved through the characterisation of a handful of bright targets.

- The characterisation of atmospheric properties in giant planets, in particular the presence of clouds, their physical and chemical properties and their evolution in time is crucial to understand the planetary long-term evolution and infer bulk compositions. Also in that case, the possibility to observe tens or hundreds of planets with Ariel to acquire spectra spanning the entire visible to infrared wavelength range is needed.

- Finally, we stress that the observation of signatures of refractory species like TiO, $\mathrm{Fe}, \mathrm{Na}$ or $\mathrm{S}$ (which may be brought into planets as $\mathrm{FeS}$ ) may yield constraints on the ice-to-rock ratios in giant exoplanets, a crucial parameter for formation models (see [73]).

From the viewpoints both of planet formation and planetary interior, of particular interest are the atmospheric compositions of giant planets with estimated bulk compositions. At present, the problem is that different types of samples (warm Jupiters' interiors and hot Jupiters' atmospheres) have been compared. There are more than ten target planets that are overlapping between the current Ariel MRS list [25] and [142]'s list, which include CoRoT-10 b, HAT-P-15 b, HAT-P-17 b, HAT-P-20 b, HAT-P-54 b, HATS-6 b, HATS-17 b, HD 17156 b, HD 80606 b, Kepler-16 b, WASP-8 b, WASP$80 \mathrm{~b}$, WASP-84 b, WASP-130 b, and WASP-132 b. We suggest that these planets are particularly interesting for understanding the origin of heavy elements of close-in gas giants and also the partitioning of heavy elements between the atmosphere and interior.

\section{Intermediate-mass planets}

\subsection{An abundant yet poorly known class of planets}

While in the Solar System there is a clear division between terrestrial and giant planets by mass and/or size, exoplanet data have taught us that although planets can be refractory or $\mathrm{H}-\mathrm{He}$ dominated, there is a non-negligible population of planets that 
have intermediate masses $\left(1-20 M_{\oplus}\right)$ and radii (1-4 $\left.R_{\oplus}\right)$, [111]. These planets represent a unique planetary class - it is not possible to simply re-scale models of the terrestrial or gas giant planets. Intermediate-mass planets can be larger versions of terrestrial planets (e.g., super-Earths) or smaller versions of giant planets (e.g., miniNeptunes) but could also be a class of planets that have different compositions than what is typically assumed, such as iron-coreless [29], carbon-rich [92, 98], waterrich [72, 81], or Ca-Al-rich [24] planets. The transition between terrestrial-like to gaseous-like (H-He dominated) planets is unclear, and at a given planetary mass a planet can belong to either of these populations [1]. This is demonstrated in Fig. 1, where we show the M-R relation of exoplanets with well-determined masses and radii.

This intermediate "overlapping" population is of great interest to the planetary community since the formation and evolutionary paths of such planets are poorly understood. This is true both for super-Earths [75] and mini-Neptunes [48, 161]. It may be possible to distinguish between dominantly rocky, icy or gaseous planets, when additional information on the age of the exoplanetary system exists and the composition of the planetary atmosphere becomes known. In Fig. 4 one can see the intermediate-mass/size planetary population. Several key questions linked to intermediate-mass exoplanets include:

- What is the atmospheric composition of intermediate-mass planets?

- Are the atmospheres of intermediate-mass exoplanets primordial?

- What are the typical compositions of intermediate-mass planets?

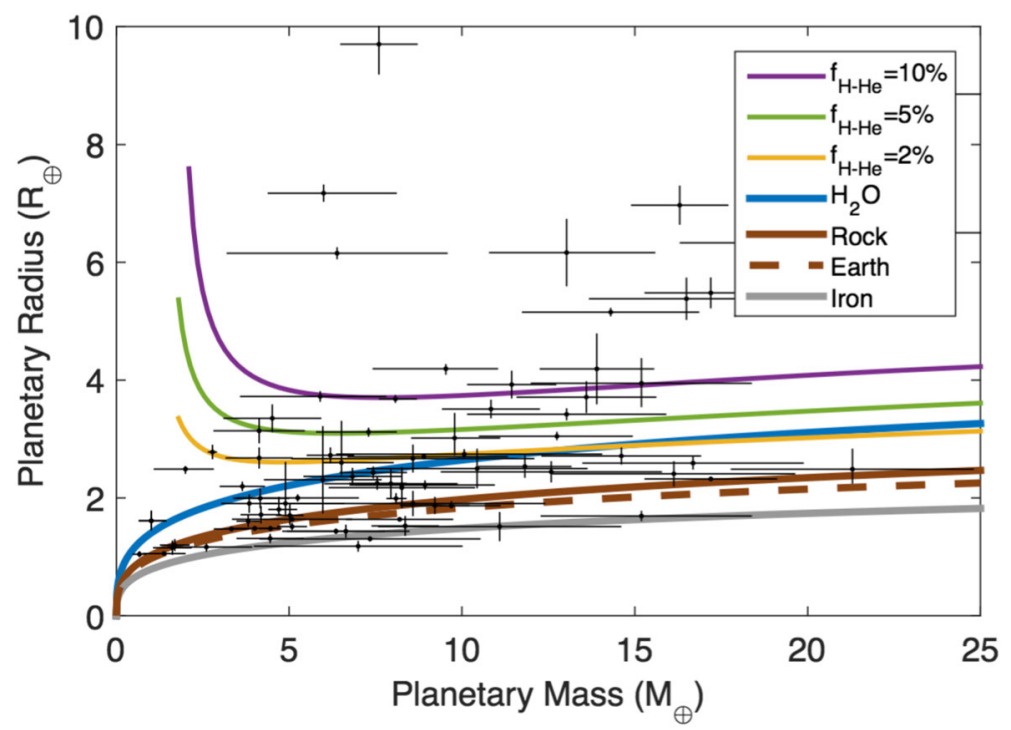

Fig. 4 Theoretical mass vs. radius relationships for planets of pure iron, rock $\left(\mathrm{MgSiO}_{3}\right)$ and water composition as well as Earth-like interior, in addition to rocky planets harboring H-He atmospheres with mass fractions of $2 \%, 5 \%$ and $10 \%$. The black dots with the error-bars show a sample of small and intermediate mass exoplanets. The figure is taken from [90] 
- Are intermediate-mass planets rich in water?

- How do the atmospheres of intermediate-mass planets interact with their deep interiors/surfaces?

Intermediate-mass planets of several Earth masses with gaseous envelopes, are expected to stay in a molten phase for giga-years [160]. The long-last interaction of the molten magma-ocean surface with the convective envelope can enrich the envelope with metals, and affect the planet atmospheric abundances [68]. Yet, the partitioning behaviour of volatiles into magma at increasingly high pressures is uncertain and requires concerted experimental effort to connect atmospheric models of extrasolar planets to petrologic data. Measurements of the atmospheric abundance of magma-ocean planets with gas envelopes by the Ariel mission will put constraints on the efficiency of the magma-envelope interaction.

Water-rich interiors are also possible scenarios for intermediate-mass planets of radii below 2.6 $R_{\oplus}$ [90]. For example, stars of very low-mass or low content of ${ }^{26} \mathrm{Al}$ are thought to host planets rich in water [84]. However, mass and radius alone cannot distinguish between gas-rich or water-rich interiors and additional constraints on atmospheric composition by Ariel are key to further reduce the degeneracy.

In the Solar System, this class of planets is only represented by Uranus and Neptune, which are poorly understood (e.g., [49, 53]). Observations of planets with similar masses and radii of Uranus and Neptune with Ariel would provide highly informative and complementary statistical information on a wide variety of planets with intermediate masses/sizes. A future mission to Uranus and Neptune (e.g., [32, 46]) would give us the keys to really understand Uranus and Neptune and reflect this knowledge on intermediate-mass exoplanets.

\subsection{Ariel observations of intermediate-mass planets}

Like gas giant planets, intermediate-mass planets are key targets to address questions linked to the composition, formation, and evolution of planets as identified in Table 1. Given their common occurrence in the galaxy (e.g., [37]), we can expect significant improvement in the characterization of this diverse and still mysterious class of planets with Ariel. In particular, key observations include:

- The transition from gas to ice giants needs to be well understood, in particular by characterizing atmospheric compositions of a variety of planets with masses $\sim 0.3 \mathrm{M}_{\mathrm{J}}$. Given the large parameter space (in terms of orbital period, composition, eccentricity, stellar properties), we envision that a large ensemble of tens or hundreds of planetary atmospheres should be characterised by Ariel. This will lead to a significant progress in our understanding of the nature of intermediate-mass planets and their formation mechanism.

- Because of their smaller mass, and therefore, lower gravity and limited atmospheric/envelope mass, intermediate-mass planets, together with Super-Earths, are crucial targets to understand atmospheric evaporation. Observations of tens to hundreds of planets with intermediate masses that orbit close to their stars class are desirable. 
- Intermediate-mass planets should show a much larger variety of atmospheric compositions than giant planets, owing to the potentially low abundance of hydrogen and helium (or conversely, the potentially high metallicity) in their atmospheres. Studies of their atmospheric compositions can be used to understand the link between ice-to-rock ratio and planet formation mechanisms, the role of clouds in planetary atmospheres, and the relations between atmospheric and bulk composition.

\section{Terrestrial planets}

Recent studies indicate rocky exoplanets are common [66]. So far, over 1000 exoplanets whose radii are less than $2 R_{\oplus}$ have been discovered. At the lower radius end of these size range (1-2 $\left.R_{\oplus}\right)$ the planet classification enters the terrestrial planet regime [65, 112]. Fulton et al. [37] suggests that there is a detection deficiency around 1.5-2 $R_{\oplus}$ for small close-in planets so that the observed limit may rather be $1.5 R_{\oplus}$.

Observations of terrestrial planets are generally restricted to radius and mass, thus the bulk density can be determined. Detailed interior structure models of terrestrial planets are obtained by combining mineral physics and average composition. Predictions for Earth-like exoplanets derived from solar system terrestrial planets are limited by the defined composition, which results in iron cores, silicate mantles and negligible but visible atmospheric mass.

During the main epoch of accretion, rocky planets likely melt entirely due to release of potential energy [28], of short-lived radioactive isotopes as in the Solar System [54, 82], and thermal blanketing of the captured nebular proto-atmosphere $[63,109]$. As a result Fe metal, which is immiscible with silicate, is able to sink to the planet's centre forming a metal core (carrying with it elements with an affinity to chemically bind to Fe, such as Ni and limited amounts of light elements, e.g., H, C, $\mathrm{N}, \mathrm{O}, \mathrm{S}, \mathrm{Si},[17,31,55,57])$. The silicate mantle left behind undergoes further differentiation, producing a crust and atmosphere. Such differentiated planets may enter a range of geodynamic regimes, of which Earth's plate tectonics is one example [127]. The geodynamic regime entered is intimately linked with a planet's thermal history, influencing whether a magnetic field develops due to core convection, the structure and stability of planetary crusts, rates of volcanism, and the efficiency of surface recycling [33]. A comprehensive review of the solar system terrestrial bodies can be found in Trønnes et al. [145].

The history of Earth, Venus, and Mars demonstrates the diversity of terrestrial planet atmospheres. The mass and composition of an atmosphere of terrestrial planets evolves through delivery of volatiles by nebular ingassing [109, 165], volatile-ice rich precursors [122], outgassing from the rocky interior [56, 63, 126], and loss to space [63]. Delivery by solid phases due to planetesimals/pebbles is expected to dominate during the early stages, and may be altered due to their internal geophysical evolution [84] that can alter the structural properties of rocky planets in a statistical fashion (Fig. 5). Outgassing occurs during magma ocean cooling [10, 56, 63, 124, 130] but can continue during a planet's life-time through volcanism (e.g., like we know it from Earth today). 


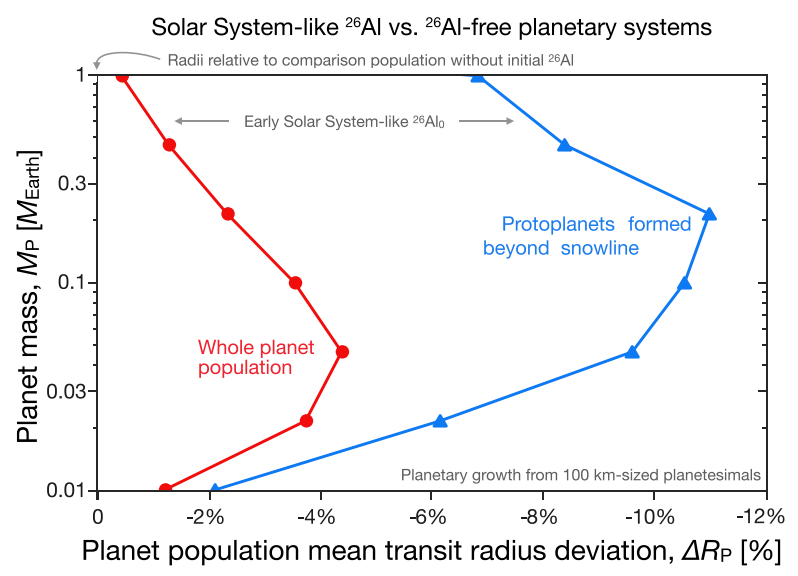

Fig. 5 Predicted deviations in $M-R$ diagram for rocky planets formed with solar system-like abundances of the short-lived radionuclide ${ }^{26} \mathrm{Al}$ compared to ${ }^{26} \mathrm{Al}$-free systems. Planets with similar or higher than Solar abundances tend to form dried-out rocky planets; planets in ${ }^{26} \mathrm{Al}$-free systems are statistically significantly enriched in water. The abundances of ${ }^{26} \mathrm{Al}$ scale with the mass of the star-forming environment of a given planetary system [83], suggesting a significant divergence in rocky planetary radii across these two regimes: planetary systems from massive star-forming regions form terrestrial planets; those from low-mass environments statistically tend to form ocean worlds with water contents on the order of $\sim 10 \%$. Figure modified from [84]

The chemistry and efficiency of outgassing/ingassing are controlled by many aspects among which some are largely unconstrained (e.g., mantle dynamics, thermal state) and some can be constrained (e.g., surface gravity, bulk composition, redox state). While small terrestrial exoplanets cannot be probed directly, their composition and evolution may be inferred from knowledge of the thickness and composition of their atmospheres as constrained by Ariel observations. However, further progress in theory is required to take full advantage of Ariel data.

Like for the gaseous planets, major challenges for understanding the planetary interior structure relate to our progress in developing structure and evolution models. We need to further investigate uncertainties associated with the assumed interior structure, which are the abundance of elements, the used equations of state, and the actual size of the planet, as well as the effect of the stellar radiation on the planet. Observations by Ariel could permit inferences and allow for constraining or discarding current model postulates, including several aspects such as implications for interior dynamics, crusts, and atmospheres.

\subsection{Bulk composition and consequences for secondary atmospheres}

Planets that form within the same proto-planetary disk can have very different volatile element budgets (e.g., [106]) but are expected to have similar budgets in relative refractory elements (e.g., [30]). The reason is that condensation fronts of refractory compounds (e.g., of $\mathrm{Al}, \mathrm{Ca}, \mathrm{Mg}, \mathrm{Si}, \mathrm{Fe}, \mathrm{Na}$ ) occur within a small region near the star, whereas condensation fronts of volatile compounds (incl. S, C, O, N, He, H) occur in a very extended region around the star (e.g., [164]). Chemical kinetics timescales 
for volatile elements also become comparable to other evolutionary timescales in the disk, adding variation to the fraction available as condensates or vapor. Therefore, relative abundances of refractory and rock-forming elements have been thought to be, to first order, identical between the host star and the planetary building blocks and eventually the planets. As a result, it is often assumed that $\mathrm{Mg} / \mathrm{Si}$ and $\mathrm{Fe} / \mathrm{Si}$ ratios of the planet bulk can be directly informed by the host star abundance [22, 149]. The majority of planet hosting stars have molar $\mathrm{Mg} / \mathrm{Si}$-ratios between 0.7 and 1.5, and molar Fe/Si-ratios between 0.5 and 1.0. Within this range, the Solar composition is average in terms of $\mathrm{Mg} / \mathrm{Si}$, but near the higher end of $\mathrm{Fe} / \mathrm{Si}$.

However, a recent study suggests that the refractory ratios of both $\mathrm{Fe} / \mathrm{Si}$ and $\mathrm{Fe} / \mathrm{Mg}$ ratios have a wider distribution in super-Earth planets compared to that of planethosting stars [116]. This finding challenges the assumption that super-Earths and the cores of mini-Neptunes can be assumed to have the same refractory composition as that of the hosting star. A recent study by [131] investigates the eleven individual systems with well-characterized Super-Earths for which host star abundances are available. It was found that only one planet does not reflect its host star abundance and is expected to have a Super-Mercury interior.

Plotnykov and Valencia [116] showed that the uncompressed density is an ideal metric to compare the planetary composition. This is different than the commonly used bulk composition that depends on both pressure-temperature regime and composition. They obtain the uncompressed density of all exoplanets that are consistent with a rocky composition (below the threshold radius for rocky planets [89]) and with mass and radius uncertainty less than $25 \%$. It appears there is a maximum enrichment in iron corresponding to an uncompressed density of $\sim 6 \mathrm{~g} \mathrm{~cm}^{-3}$.

Fortunately, the Ariel mission will provide reliable and homogeneous stellar abundances (Danielski et al. in prep., Ariel Stellar Characterisation WG). In combination with internal structure models we will be able to test the primordial origin hypothesis for solid planets, determine if there is indeed a maximum iron enrichment possible from formation, and put key constraints on the bulk abundance of terrestrial exoplanets.

The bulk rock composition of planets also has direct consequences on the planetary evolution of and the secondary atmosphere. The planetary bulk composition influences tectonic processes that allow volatiles to ingas from the atmosphere to the mantle [150] and outgassing through volcanism [23]. Furthermore, the bulk rock composition influences whether exoplanet mantles convect in a single layer or experience double-layered convection [136]. This has direct implications for the exchange of volatile between reservoirs. A comprehensive study that rigorously investigates the effects of bulk rock composition on melting and outgassing is still lacking and further theoretical efforts are needed to better understand the link between bulk rock composition and atmosphere evolution and chemistry.

Finally, a crucial aspect that determines the chemistry of a secondary atmosphere is the mantle oxidation state. Under reducing conditions, the outgassing of $\mathrm{H}_{2}$ and $\mathrm{CO}$ is favoured, while oxidising conditions favour $\mathrm{H}_{2} \mathrm{O}$ and $\mathrm{CO}_{2}$ to outgas $[21,56]$. Changing how reducing an atmosphere is has important implications for prebiotic chemistry [120] and climate [167]. 


\subsection{Interior structure and dynamics}

The system's redox state of terrestrial planets is of high importance. This is because it determines the core-mantle fraction, as well as the secondary atmosphere composition, which can be dominated either by $\mathrm{H}_{2} \mathrm{O}$ and $\mathrm{CO}_{2}$ or $\mathrm{H}_{2}$ and $\mathrm{CH}_{4}$ for example. The availability of oxygen in the exoplanetary system and during planetary accretion, allows for core formation due to the oxidation of the mantle (e.g., [36]).

Super-Earths cover a mass range of up to $10 M_{\oplus}$, although early studies speculate interior structures with Earth-like mantle-core fraction to up to $20 M_{\oplus},[58,111]$. Given the solar system planet composition range, there is likely an upper limit of super-Earth radius for an Earth-like mean density [67], which is at most $2 R_{\oplus}$ [90]. Scaling Earth's structure to larger objects, Tackley et al. [139] investigated the potential range of interior dynamics and surface tectonic expressions (stagnant, episodic, mobile), finding that convection still takes place in the interior of large super-Earths but that it can be sluggish, and confirming earlier findings (e.g., [151]) that larger planets are more likely to display plate tectonics. With possibly only slight variations in the parameter set, however, it has also been found that size may not be important or increased size rather hinder the development of plate tectonics ([33], and references therein). Conditions can vary due to the variations in the different systems, which include surface temperature (regulated by atmosphere and distance to the central star), internal heating (related to radiogenic elements and/or tidal dissipation), differences in yield stress (due to composition, particularly variations in water content of the rock crystal structure).

Several studies provide a phase diagram that suggests a complex relation of these parameters for the evolution of surface tectonic regimes (e.g., [110]); however, such studies neglected magmatism and crustal production, which can have a first order effect on the tectonic regime, particularly during early, hot phases [87, 88]. Thus, there are not only limitations in the numerical implementation of geological processes, but actual lack of knowledge on for example material behaviour under high pressures, when the mixtures are more complex, which hinders more reliable determination of interior structure, composition and evolution of the terrestrial exoplanets.

The orbital setting of close-in planets suggests that they are tidally locked, so that interior dynamics and surface tectonics may differ between the star facing and its opposite side. Several studies investigated the stellar side melting of surface materials and the formation of hemispherical magma oceans [155]. This molten surface adds a fourth tectonic regime besides, stagnant, episodic or mobile lid known from our solar system. Given the potential of an hemispherical protracted magma ocean, exoplanet interior dynamics may not follow any of the solar system regimes. Observations and phase maps of rocky super-Earths can improve our understanding of the physical mechanisms driving tectonic changes on rocky planets. Furthermore, different regimes of interior-atmosphere exchange relative to Earth and the solar system terrestrial planets will guide our development of more robust geophysical models of the thermo-chemical evolution of rocky proto-planets and their emerging atmospheres. 
Using constraints from such phase maps (e.g., [20, 71]), interior dynamical modelling provide insight to interior states of observed super-Earths in order to relate the tectonic, degassing, and atmospheric evolution (Fig. 6). Phenomena related to these hemispherical tectonic regimes may, however, be short-lived, because the planet may continuously reorient due to true polar wander [78], and then such a hemispherical difference may be small.

\subsection{Time dependence of the interior state and implications for atmospheres}

The potential for a terrestrial planet to form (and hold) an atmosphere depends on is mass, as well as the distance to the host star [65]. The atmospheric composition depends on the evolutionary stage (age) of the planetary system and the interior structure and interior dynamics generating a magnetic field for the individual planet [9, 38].

Earth's atmosphere has been profoundly shaped by the presence of life (e.g., the release of oxygen by photosynthesising plants, and sequestration of atmospheric carbon into rocks). The detection of water in the atmosphere may be a requirement for life, but it is insufficient to demonstrate life being present in other systems. At the earliest stages of planet formation, potentially primordial $(\mathrm{H}-\mathrm{He})$ composition dominates the atmospheres, but water may be present [63]. Water or steam atmospheres could cause protracted magma oceans and delay cooling of the surface [8, 10], prohibiting the formation of lids (lithosphere).
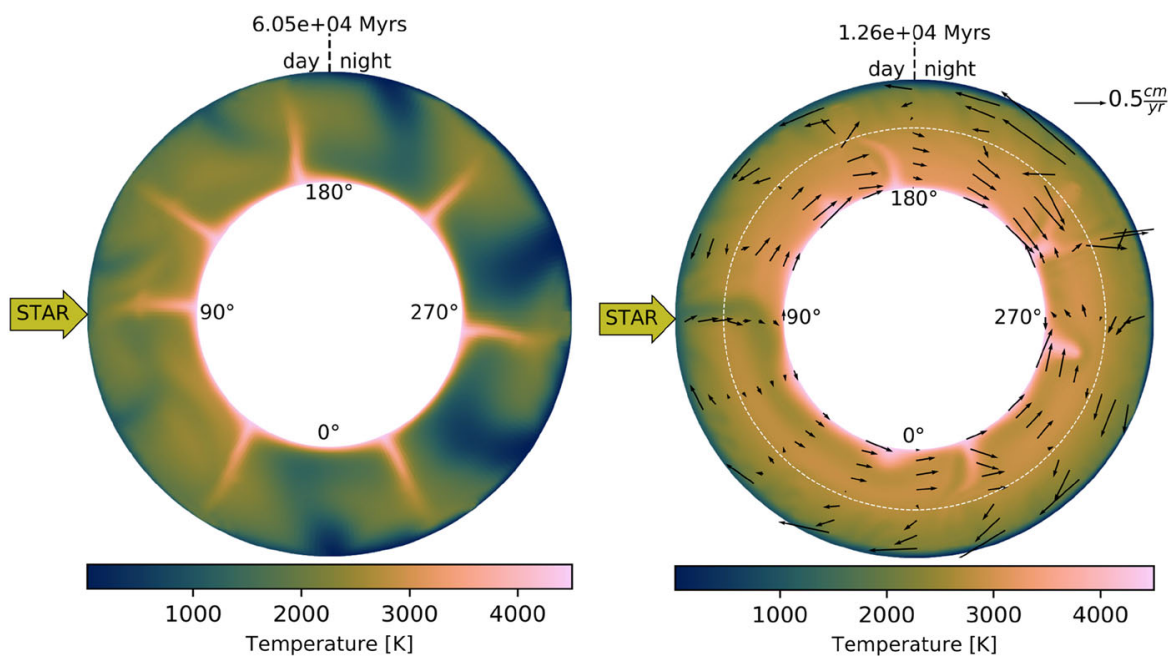

Fig. 6 Model of the geodynamic and tectonic state of the interior of LHS 3844b, assuming isothermal surface boundary conditions according to the phase maps from Kreidberg et al. [71]. The figure shows the temperature field with a weak (left) and a strong (right) surface layer, with a substellar surface temperature of $1000 \mathrm{~K}$ and antistellar surface temperature of $20 \mathrm{~K}$. The distribution of plumes in the hemispheric tectonics regime (right) beneath the substellar point and the distribution of largely molten regions points to a tectonic regime not observed in the Solar System and may provide vital clues as to the potential variability of tectonic states across the rocky exoplanet census. Figure adapted from Meier et al. [94] 
The extent and mass of an atmosphere has a significant impact on the proportions of the solid and gaseous parts of terrestrial planets, and thus on the observed density. The evolution of terrestrial planet atmospheres and speciation of volcanic gases is temperature dependent $[36,56]$. Degassing due to volcanism enriches the atmosphere in water, ammonia, methane and/or carbon dioxide, while the primordial atmosphere is lost to space, if the planet is too small, too hot (interior or stellar insulation), or does not have a protecting magnetic field. The latter requires a convecting metal core (terrestrial planets) or, in instances where these objects are similar to giant planet's satellites, subsurface saline oceans. The persistence of a magnetic field of the small icy satellites in the solar system requires external heat sources such as tidal interaction with the planet. The persistence of a strong magnetic field of rocky planets is often linked to active plate tectonics. High or low surface temperatures, or climate, has been suggested an important boundary condition of whether plate tectonics would occur. In turn plate tectonic processes have been suggested to moderate climate to be temperate, and allow for cooling of the interior to sustain mantle and core convection to generate the magnetic field.

There are large uncertainties regarding the initiation and sustainability of plate tectonics ([33], and references therein), which relate to the unknown composition and rheologic properties of the material and the planet, and it remains unclear whether there is a size dependency on the propensity to plate tectonics and similarly the magnetic field strength.

Close-in terrestrial planet are expected to lose their atmosphere due to the stellar radiation pressure. However, if atmospheric gases would be detected by Ariel, a dominance of $\mathrm{H}_{2}$ and $\mathrm{CH}_{4}$ suggest active degassing, while the dominance of $\mathrm{H}_{2} \mathrm{O}$ and $\mathrm{CO}_{2}$ may indicate the presence of an atmosphere protecting strong magnetic field, including possible plate tectonics in old systems.

\subsection{Constraints on magma composition of hot rocky super-Earths from atmospheric measurements}

Over 1000 exoplanets with radii smaller than $2 R_{\oplus}$ have been discovered. About half of these planets have substellar-point equilibrium temperatures $T_{\text {irr }}$ high enough ( $\gtrsim 1500 \mathrm{~K}$ ) for rock to melt and vaporise (see Fig. 7), which include 55 Cnc e whose substellar-point temperature is estimated to be about $2700 \mathrm{~K}$, with zero planetary albedo. Most of the close-in small exoplanets, if they are rocky, are probably planets that have lost their primordial hydrogen-rich atmosphere due to photo-evaporation. The closer the planets are to the star, they may be bare of all volatiles, but their rocky surfaces are thought to be molten and they have secondary atmospheres vaporised from the magma due to the high temperatures. We call these rocky planets hot rocky super-Earths (hereafter HRSEs).

Depending on their evolutionary pathways, starting as solar system type terrestrial planets or inwards migrated mini-Neptunes from beyond the ice line, they may be rocky planets, but their interior structures and compositions are mostly unknown at present. Several theoretical studies argue for the presence of not only terrestrial planets with similar interiors to those of solar system's rocky planets, but also (iron-) coreless planets [29], carbon-rich planets [92, 98], water-rich planets [84, 170] or 
Ca-Al-rich planets [24]. To determine the interior composition of rocky exoplanet, atmospheric observations of HRSEs in particular allow direct constraints, because their secondary atmospheres are likely composed of materials directly vaporised from their magma ocean.

If HRSEs are dry, they likely have atmospheres composed of rocky materials such as $\mathrm{Na}, \mathrm{K}, \mathrm{Fe}, \mathrm{Si}, \mathrm{O}, \mathrm{O}_{2}$ and $\mathrm{SiO}[64,96,128]$. On the other hand, if HRSEs have remaining volatile elements such as $\mathrm{H}, \mathrm{C}, \mathrm{N}, \mathrm{S}$ and $\mathrm{Cl}$, they likely have atmospheres composed mainly of $\mathrm{H}_{2} \mathrm{O}$ and/or $\mathrm{CO}_{2}$ with rocky vapours such as $\mathrm{Na}$ and $\mathrm{SiO}$ [10, 129]. Or by $\mathrm{HCN}$ in case of $\mathrm{N}$-dominated atmospheres [95, 171] Generally, we call the former case a mineral atmosphere and the latter case a steam atmosphere. Thus, detection of rocky vapour would provide a definitive piece of evidence for HRSEs and their surface composition, including indications of their deeper structure. Identifying the atmospheric constituents could give constraints on the bulk composition and formation processes of the HRSEs, but this requires an atmosphere with low cloud coverage (compare report of the Ariel Chemical Working Group). Currently, no studies on the vaporised atmospheres of molten coreless, carbon-rich, or Ca-Al-rich planets exist, and we suggest that this topic should be addressed in future research.

Ariel observations will provide clues for interior structure of HRSEs and suffice to distinguish a mineral atmosphere from a cloud-free, hydrogen-rich or water-rich atmosphere, while planets covered completely with thick clouds or with no atmosphere show flat spectra, which are similar to that of mineral atmospheres, detection

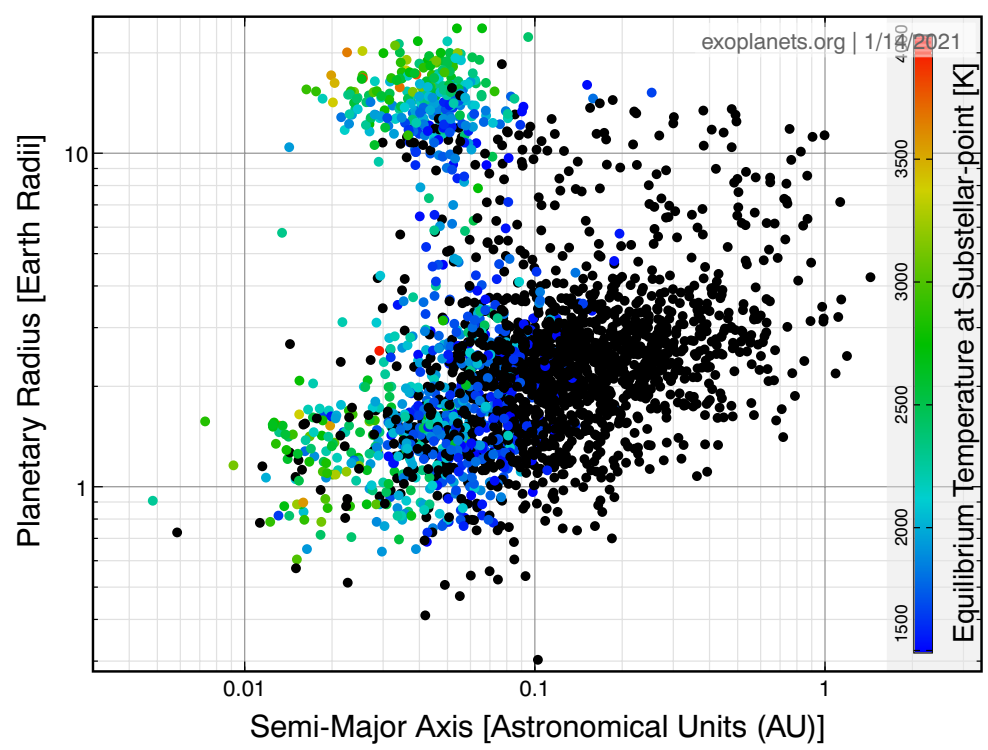

Fig. 7 Two-dimensional distribution of discovered exoplanet size and orbit. The colour contours shows the substellar-point equilibrium temperatures with zero planetary albedo larger than $1500 \mathrm{~K}$. The data has been taken from an open exoplanet catalogue database (http://exoplanets.org) 
of $\mathrm{Na}(0.6 \mu \mathrm{m})$ and $\mathrm{K}(0.8 \mu \mathrm{m})$ with ground-based telescopes would be helpful to distinguish a mineral atmosphere from other possibilities. Therefore, Ariel would provide information on the absence or presence of volatile elements in molten surfaces of HRSEs.

Using 55 Cancri e as an example for the discussion, composition is a matter of speculation and needs observational constraints, but the tentative detection of hydrogen [146] indicates that some concepts may be oversimplified. The closeness of a planet to a star suggests that all volatile components should have been evaporated, if its interior mixing had been fast enough to rapidly supply these materials to the atmosphere [74, 86]. Numerical studies [99, 139], however, predict that mantle convection of a super-Earth sized terrestrial planet can be very slow. Therefore, HRSEs' magma can retain volatile materials due to the weak interior-atmosphere interaction. The presence of abundant $\mathrm{H}$ but an absence of water vapour might suggest that the atmosphere is vaporised from reduced magma retaining hydrogen and also $\mathrm{CO}$ and $\mathrm{SiO}$ [129]. Therefore, the detection of these gas species would lead to ascertaining the reduced magma composition and mantle convection slow enough to retain hydrogen in the interior.

\subsection{Ariel observations of terrestrial planets}

Like the other planetary types, terrestrial exoplanets are of particular interest. With the aim to a better understand the diversity of terrestrial planet interiors and interactions with their atmosphere, transiting planets with measured masses and radii with high accuracy are ideal targets for atmospheric follow-up characterisation. This information can then be used to infer the relation between interior, formation and evolution as indicated in Table 1. Although the Ariel mission is not focused on small planets, it may help to address the following questions:

- Ariel measurements will be used to determine the frequency of terrestrial planets (i.e., super-Earths) with significant atmospheres.

- Identifying metal-rich (Mercury-like) and Earth-like planets is important for our understanding of small planets and their potential habitability. Therefore spectra of tens of small planets could help to better characterize these planets.

- The diversity of potential surface compositions of terrestrial planets is yet to be determined. Ariel could provide insight on the surface composition of many small planets orbiting close to their stars.

- Ariel will also address the issue of atmosphere escape in super-Earths. This can be used to indirectly indicate the existence of magnetic fields that prevent atmospheric loss.

Planets that are included in the Ariel MRS list of [25] and that are of particular interest in this regard include 55Cnc e, GJ1132 b, GJ9827 b, HD219134 b, Kepler$138 \mathrm{~b}$ and d, LHS1140 b and c, and the seven Trappist planets b-h. Planets without measured masses are also of interest, especially when their stellar abundances are determined. Such planets include GJ9827 c, Kepler-444b, c, d, e, HD3167b, K2$129 \mathrm{~b}$. 


\section{Summary}

Exoplanet characterisation is a key goal of exoplanet science. Measurements of the planetary mass and radius alone are insufficient to uniquely determine the planetary composition. Ariel's measurements of the atmospheric composition can break some of the degeneracy in determining the planetary compositions and improve our understanding of planets.

The statistical evaluation of the various atmosphere types can assist us to discriminate among different formation and evolutionary pathways. The mission's results will allow us to promote/dismiss current and upcoming theoretical models using observations. Linking the atmospheric composition with the bulk composition, and using the information from specific elements to further constrain the composition, evolution and formation of planets is challenging and yet to be determined. Clearly, progress in theory is required in order to take full advantage of the upcoming data. The many targets of Ariel which include planets with various masses, host stars, and orbital properties will provide a wide view of the characteristics of exoplanets and on the connection between atmospheric and bulk composition.

Finally, the Ariel mission is expected to significantly improve our understanding of the interplay between planet formation, evolution and internal structure.

Acknowledgements We thank the two anonymous referees for valuable comments. RH acknowledges support from the Swiss National Science Foundation (SNSF) via grant 200020_188460. SCW is supported by the Research Council of Norway through its Centers of Excellence funding scheme, project number 223272 (CEED). CD acknowledges support from the Swiss National Science Foundation under grant PZ00P2_174028. TL received funding from the Simons Collaboration on the Origins of Life (grant no. 611576) and the SNSF (grant no. P2EZP2-178621). Parts of the work presented here was conducted within the framework of the National Centre for Competence in Research PlanetS (grant no. 51NF40141881 ) supported by the Swiss National Science Foundation. This research has made use of the Exoplanet Orbit Database and the Exoplanet Data Explorer at exoplanets.org.

Funding Open Access funding provided by Universität Zürich.

Open Access This article is licensed under a Creative Commons Attribution 4.0 International License, which permits use, sharing, adaptation, distribution and reproduction in any medium or format, as long as you give appropriate credit to the original author(s) and the source, provide a link to the Creative Commons licence, and indicate if changes were made. The images or other third party material in this article are included in the article's Creative Commons licence, unless indicated otherwise in a credit line to the material. If material is not included in the article's Creative Commons licence and your intended use is not permitted by statutory regulation or exceeds the permitted use, you will need to obtain permission directly from the copyright holder. To view a copy of this licence, visit http://creativecommons.org/licenses/by/4.0/.

\section{References}

1. Adams, E.R., Seager, S., Elkins-Tanton, L.: Ocean planet or thick atmosphere: on the massradius relationship for solid exoplanets with massive atmospheres. ApJ 673(2), 1160-1164 (2008). https://doi.org/10.1086/524925. arXiv:0710.4941

2. Baraffe, I., Chabrier, G., Barman, T.S., Allard, F., Hauschildt, P.H.: Evolutionary models for cool brown dwarfs and extrasolar giant planets. The case of HD 209458. A\&A 402, 701-712 (2003). https://doi.org/10.1051/0004-6361:20030252. arXiv:0302293 
3. Baraffe, I., Chabrier, G., Barman, T.: Structure and evolution of super-Earth to super-Jupiter exoplanets. I. Heavy element enrichment in the interior. A\&A 482(1), 315-332 (2008). https:// doi.org/10.1051/0004-6361:20079321. arXiv:0802.1810

4. Batygin, K., Stevenson, D.J.: Inflating hot Jupiters with ohmic dissipation. ApJ1 714, L238-L243 (2010). https://doi.org/10.1088/2041-8205/714/2/L238. arXiv:1002.3650

5. Bodenheimer, P., Pollack, J.B.: Calculations of the accretion and evolution of giant planets: the effects of solid cores. Icarus 67(3), 391-408 (1986). https://doi.org/10.1016/0019-1035(86)90122-3

6. Bodenheimer, P., Lin, D.N.C., Mardling, R.A.: On the tidal inflation of short-period extrasolar planets. ApJ 548, 466-472 (2001)

7. Bodenheimer, P., Stevenson, D.J., Lissauer, J.J., D'Angelo, G.: New formation models for the kepler36 system. ApJ 868(2), 138 (2018). https://doi.org/10.3847/1538-4357/aae928. arXiv:1810.07160

8. Bonati, I., Lichtenberg, T., Bower, D.J., Timpe, M.L., Quanz, S.P.: Direct imaging of molten protoplanets in nearby young stellar associations. A\&A 621, A125 (2019). https://doi.org/10.1051/ 0004-6361/201833158. arXiv:1811.07411

9. Boujibar, A., Driscoll, P., Fei, Y.: Super-Earth internal structures and initial thermal states. J. Geophys. Res. (Planets) 125(5), e06124 (2020). https://doi.org/10.1029/2019JE006124

10. Bower, D.J., Kitzmann, D., Wolf, A.S., Sanan, P., Dorn, C., Oza, A.V.: Linking the evolution of terrestrial interiors and an early outgassed atmosphere to astrophysical observations. Astron. Astrophys. 631, A103 (2019). https://doi.org/10.1051/0004-6361/201935710. arXiv:1904.08300

11. Brouwers, M.G., Vazan, A., Ormel, C.W.: How cores grow by pebble accretion. I. Direct core growth. A\&A 611, A65 (2018). https://doi.org/10.1051/0004-6361/201731824. arXiv:1708.05392

12. Burrows, A., Guillot, T., Hubbard, W.B., Marley, M.S., Saumon, D., Lunine, J.I., Sudarsky, D.: On the radii of close-in giant planets. ApJ1 534(1), L97-L100 (2000). https://doi.org/10.1086/312638. arXiv:0003185

13. Burrows, A., Hubeny, I., Budaj, J., Hubbard, W.B.: Possible solutions to the radius anomalies of transiting giant planets. ApJ 661(1), 502-514 (2007). https://doi.org/10.1086/514326. arXiv:0612703

14. Chabrier, G., Baraffe, I.: Heat transport in giant (Exo)planets: a new perspective. ApJ1 661(1), L81L84 (2007). https://doi.org/10.1086/518473. arXiv:0703755

15. Chabrier, G., Gallardo, J., Baraffe, I.: Evolution of low-mass star and brown dwarf eclipsing binaries. A\&A 472(2), L17-L20 (2007). https://doi.org/10.1051/0004-6361:20077702. arXiv:0707.1792

16. Chatterjee, S., Chen, H.: Effects of planetesimal accretion on the thermal and structural evolution of sub-Neptunes. ApJ 852(1), 58 (2018). https://doi.org/10.3847/1538-4357/aa9e05. arXiv:1708.05366

17. Dalou, C., Füri, E., Deligny, C., Piani, L., Caumon, M.C., Laumonier, M., Boulliung, J., Edén, M.: Redox control on nitrogen isotope fractionation during planetary core formation. Proc. Natl. Acad. Sci. 116(29), 14485-14494 (2019). https://doi.org/10.1073/pnas.1820719116

18. Debras, F., Chabrier, G.: New models of Jupiter in the context of Juno and Galileo. ApJ 872(1), 100 (2019a). https://doi.org/10.3847/1538-4357/aaff65. arXiv:1901.05697

19. Demory, B.O., Seager, S.: Lack of inflated radii for Kepler giant planet candidates receiving modest stellar irradiation. ApJs 197(1), 12 (2011). https://doi.org/10.1088/0067-0049/197/1/12. arXiv: 1110.6180

20. Demory, B.O., Gillon, M., de Wit, J., Madhusudhan, N., Bolmont, E., Heng, K., Kataria, T., Lewis, N., Hu, R., Krick, J., Stamenković, V., Benneke, B., Kane, S., Queloz, D.: A map of the large day-night temperature gradient of a super-Earth exoplanet. Nature 532(7598), 207-209 (2016). https://doi.org/10.1038/nature17169. arXiv:1604.05725

21. Deng, J., Du, Z., Karki, B.B., Ghosh, D.B., Lee, K.K.M.: A magma ocean origin to divergent redox evolutions of rocky planetary bodies and early atmospheres. Nat. Commun. 11, 2007 (2020). https://doi.org/10.1038/s41467-020-15757-0

22. Dorn, C., Khan, A., Heng, K., Connolly, J.A.D., Alibert, Y., Benz, W., Tackley, P.: Can we constrain the interior structure of rocky exoplanets from mass and radius measurements? A\&A 577, A83 (2015). https://doi.org/10.1051/0004-6361/201424915. arXiv:1502.03605

23. Dorn, C., Noack, L., Rozel, A.: Outgassing on stagnant-lid super-earths. Astron. Astrophys. 614, A18 (2018)

24. Dorn, C., Harrison, J.H., Bonsor, A., Hands, T.O.: A new class of super-earths formed from hightemperature condensates: Hd219134 b, $55 \mathrm{cnc}$ e, wasp-47 e. Mon. Not. R. Astron. Soc. 484(1), 712727 (2019)

25. Edwards, B., Mugnai, L., Tinetti, G., Pascale, E., Sarkar, S.: An updated study of potential targets for Ariel. AJ 157(6), 242 (2019). https://doi.org/10.3847/1538-3881/ab1cb9. arXiv:1905.04959 
26. Ehrenreich, D., Lovis, C., Allart, R., Zapatero Osorio, M.R., Pepe, F., Cristiani, S., Rebolo, R., Santos, N.C., Borsa, F., Demangeon, O., Dumusque, X., González Hernández, J.I., Casasayas-Barris, N., Ségransan, D., Sousa, S., Abreu, M., Adibekyan, V., Affolter, M., Allende Prieto, C., Alibert, Y., Aliverti, M., Alves, D., Amate, M., Avila, G., Baldini, V., Bandy, T., Benz, W., Bianco, A., Bolmont, É., Bouchy, F., Bourrier, V., Broeg, C., Cabral, A., Calderone, G., Pallé, E., Cegla, H.M., Cirami, R., Coelho, J.M.P., Conconi, P., Coretti, I., Cumani, C., Cupani, G., Dekker, H., Delabre, B., Deiries, S., D’Odorico, V., Di Marcantonio, P., Figueira, P., Fragoso, A., Genolet, L., Genoni, M., Génova Santos, R., Hara, N., Hughes, I., Iwert, O., Kerber, F., Knudstrup, J., Land oni, M., Lavie, B., Lizon, J.L., Lendl, M., Lo Curto, G., Maire, C., Manescau, A., Martins, C.J.A.P., Mégevand, D., Mehner, A., Micela, G., Modigliani, A., Molaro, P., Monteiro, M., Monteiro, M., Moschetti, M., Müller, E., Nunes, N., Oggioni, L., Oliveira, A., Pariani, G., Pasquini, L., Poretti, E., Rasilla, J.L., Redaelli, E., Riva, M., Santana Tschudi, S., Santin, P., Santos, P., Segovia Milla, A., Seidel, J.V., Sosnowska, D., Sozzetti, A., Spanò, P., Suárez Mascareño, A., Tabernero, H., Tenegi, F., Udry, S., Zanutta, A., Zerbi, F.: Nightside condensation of iron in an ultrahot giant exoplanet. Nature 580(7805), 597-601 (2020). https://doi.org/10.1038/s41586-020-2107-1. arXiv:2003.05528

27. Eistrup, C., Walsh, C., van Dishoeck, E.F.: Setting the volatile composition of (exo)planetbuilding material. Does chemical evolution in disk midplanes matter? A\&A 595, A83 (2016). https://doi.org/10.1051/0004-6361/201628509. arXiv:1607.06710

28. Elkins-Tanton, L.T.: Magma oceans in the inner solar system. Annu. Rev. Earth Planet. Sci. 40(1), 113-139 (2012). https://doi.org/10.1146/annurev-earth-042711-105503

29. Elkins-Tanton, L.T., Seager, S.: Coreless terrestrial exoplanets. ApJ 688(1), 628-635 (2008). https://doi.org/10.1086/592316. arXiv:0808.1908

30. Elser, S., Meyer, M.R., Moore, B.: On the origin of elemental abundances in the terrestrial planets. Icarus 221(2), 859-874 (2012)

31. Fischer, R.A., Cottrell, E., Hauri, E., Lee, K.K.M., Le Voyer, M.: The carbon content of earth and its core. Proc. Natl. Acad. Sci. 117(16), 8743-8749 (2020). https://doi.org/10.1073/pnas.1919930117. https://www.pnas.org/content/117/16/8743, https://www.pnas.org/content/117/16/8743.full.pdf

32. Fletcher, L.N., Helled, R., Roussos, E., Jones, G., Charnoz, S., André, N., Andrews, D., Bannister, M., Bunce, E., Cavalié, T., Ferri, F., Fortney, J., Grassi, D., Griton, L., Hartogh, P., Hueso, R., Kaspi, Y., Lamy, L., Masters, A., Melin, H., Moses, J., Mousis, O., Nettleman, N., Plainaki, C., Schmidt, J., Simon, A., Tobie, G., Tortora, P., Tosi, F., Turrini, D.: Ice giant systems: the scientific potential of orbital missions to Uranus and Neptune. Planet. Space Sci. 191, 105030 (2020). https://doi.org/10.1016/j.pss.2020.105030. arXiv:1907.02963

33. Foley, B.J., Driscoll, P.E.: Whole planet coupling between climate, mantle, and core: implications for rocky planet evolution. Geochem., Geophys. Geosyst. 17(5), 1885-1914 (2016). https://doi.org/10.1002/2015GC006210. arXiv:1711.06801

34. Fortney, J.J., Hubbard, W.B.: Phase separation in giant planets: inhomogeneous evolution of Saturn. Icarus 164, 228-243 (2003)

35. Fortney, J.J., Ikoma, M., Nettelmann, N., Guillot, T., Marley, M.S.: Self-consistent model atmospheres and the cooling of the solar system's giant planets. ApJ 729(1), 32 (2011). https://doi.org/ 10.1088/0004-637X/729/1/32. arXiv:1101.0606

36. Frost, D.J., McCammon, C.A.: The redox state of Earth's mantle. Annu. Rev. Earth Planet. Sci. 36, 389-420 (2008). https://doi.org/10.1146/annurev.earth.36.031207.124322

37. Fulton, B.J., Petigura, E.A., Howard, A.W., Isaacson, H., Marcy, G.W., Cargile, P.A., Hebb, L., Weiss, L.M., Johnson, J.A., Morton, T.D., Sinukoff, E., Crossfield, I.J.M., Hirsch, L.A.: The california-kepler survey. iii. A gap in the radius distribution of small planets. AJ 154, 109 (2017). arXiv: 1703.10375

38. Gaidos, E., Conrad, C.P., Manga, M., Hernlund, J.: Thermodynamic limits on magnetodynamos in rocky exoplanets. ApJ 718(2), 596-609 (2010). https://doi.org/10.1088/0004-637X/718/2/596. arXiv: 1005.3523

39. Ginzburg, S., Sari, R.: Hot-Jupiter inflation due to deep energy deposition. ApJ 803(2), 111 (2015). https://doi.org/10.1088/0004-637X/803/2/111. arXiv:1501.02087

40. Ginzburg, S., Sari, R.: Extended heat deposition in hot Jupiters: application to ohmic heating. ApJ 819(2), 116 (2016). https://doi.org/10.3847/0004-637X/819/2/116. arXiv:1511.00135

41. Guillot, T.: Interior of giant planets inside and outside the solar system. Science 286, $72-77$ (1999). https://doi.org/10.1126/science.286.5437.72

42. Guillot, T.: The interiors of giant planets: models and outstanding questions. Annu. Rev. Earth Planet. Sci. 33, 493-530 (2005). arXiv:0502068 
43. Guillot, T., Showman, A.P.: Evolution of "51 pegasus b-like" planets. A\&A 385, 156-165 (2002). arXiv:astro-ph/0202234

44. Guillot, T., Burrows, A., Hubbard, W.B., Lunine, J.I., Saumon, D.: Giant planets at small orbital distances. ApJ1 459, L35-L39 (1996). arXiv:astro-ph/9511109

45. Guillot, T., Santos, N.C., Pont, F., Iro, N., Melo, C., Ribas, I.: A correlation between the heavy element content of transiting extrasolar planets and the metallicity of their parent stars. A\&A 453(2), L21-L24 (2006). https://doi.org/10.1051/0004-6361:20065476. arXiv:astro-ph/0605751

46. Guillot, T., Fortney, J., Rauscher, E., Marley, M.S., Parmentier, V., Line, M., Wakeford, H., Kaspi, Y., Helled, R., Ikoma, M., Knutson, H., Menou, K., Valencia, D., Durante, D., Ida, S., Bolton, S.J., Li, C., Stevenson, K.B., Bean, J., Cowan, N.B., Hofstadter, M.D., Hueso, R., Leconte, J., Li, L., Mordasini, C., Mousis, O., Nettelmann, N., Soderlund, K., Wong, M.H.: Keys of a mission to Uranus or Neptune, the closest ice giants. arXiv:2012.09863 (2020)

47. Helled, R.: The interiors of Jupiter and Saturn, p 175. https://doi.org/10.1093/acrefore/9780190 647926.013.175 (2019)

48. Helled, R., Bodenheimer, P.: The formation of Uranus and Neptune: challenges and implications for intermediate-mass exoplanets. ApJ 789(1), 69 (2014). https://doi.org/10.1088/0004-637X/789/1/69. arXiv: 1404.5018

49. Helled, R., Fortney, J.J.: The interiors of Uranus and Neptune: current understanding and open questions. Phil Trans R Soc A 378, 20190474 (2020). https://doi.org/10.1098/rsta.2019.0474

50. Helled, R., Lunine, J.: Measuring Jupiter's water abundance by Juno: the link between interior and formation models. MNRAS 441(3), 2273-2279 (2014). https://doi.org/10.1093/mnras/stu516. arXiv: 1403.2891

51. Helled, R., Stevenson, D.: The fuzziness of giant planets' cores. ApJl 840(1), L4 (2017). https://doi.org/10.3847/2041-8213/aa6d08. arXiv:1704.01299

52. Helled, R., Bodenheimer, P., Podolak, M., Boley, A., Meru, F., Nayakshin, S., Fortney, J.J., Mayer, L., Alibert, Y., Boss, A.P.: Giant planet formation, evolution, and internal structure. In: Beuther, H., Klessen, R.S., Dullemond, C.P., Henning, T. (eds.) Protostars and Planets VI, p. 643 (2014). https://doi.org/10.2458/azu_uapress_9780816531240-ch028. arXiv:1311.1142

53. Helled, R., Nettelmann, N., Guillot, T.: Uranus and Neptune: origin, evolution and internal structure. Space Sci. Rev. 216(3), 38 (2020). https://doi.org/10.1007/s11214-020-00660-3. arXiv:1909.04891

54. Hevey, P.J., Sanders, I.S.: A model for planetesimal meltdown by ${ }^{26} \mathrm{Al}$ and its implications for meteorite parent bodies. Meteorit. Planet. Sci. 41(1), 95-106 (2006). https://doi.org/10.1111/ j.1945-5100.2006.tb00195.x

55. Hirose, K., Tagawa, S., Kuwayama, Y., Sinmyo, R., Morard, G., Ohishi, Y., Genda, H.: Hydrogen limits carbon in liquid iron. Geophys. Res. Lett. 46(10), 5190-5197 (2019). https://doi.org/10.1029/ 2019GL082591

56. Hirschmann, M.M.: Magma ocean influence on early atmosphere mass and composition. Earth Planet. Sci. Lett. 341, 48-57 (2012). https://doi.org/10.1016/j.eps1.2012.06.015

57. Hirschmann, M.M.: Constraints on the early delivery and fractionation of Earth's major volatiles from $\mathrm{C} / \mathrm{H}, \mathrm{C} / \mathrm{N}$, and C/S ratios. Am. Mineral. 101(3), 540-553 (2016). https://doi.org/10.2138/ am-2016-5452

58. Howe, A.R., Burrows, A., Verne, W.: Mass-radius relations and core-envelope decompositions of super-Earths and sub-Neptunes. ApJ 787(2), 173 (2014). https://doi.org/10.1088/0004-637X/ 787/2/173. arXiv:1402.4818

59. Huang, X., Cumming, A.: Ohmic dissipation in the interiors of hot Jupiters. ApJ 757(1), 47 (2012). https://doi.org/10.1088/0004-637X/757/1/47. arXiv:1207.3278

60. Hubbard, W.B.: The Jovian surface condition and cooling rate. Icarus 30, 305-310 (1977)

61. Iaroslavitz, E., Podolak, M.: Atmospheric mass deposition by captured planetesimals. Icarus 187, 600-610 (2007). https://doi.org/10.1016/j.icarus.2006.10.008

62. Ikoma, M., Guillot, T., Genda, H., Tanigawa, T., Ida, S.: On the Origin of HD 149026b. ApJ 650(2), 1150-1159 (2006). https://doi.org/10.1086/507088. arXiv:astro-ph/0607212

63. Ikoma, M., Elkins-Tanton, L., Hamano, K., Suckale, J.: Water partitioning in planetary embryos and protoplanets with magma oceans. Space Sci Rev 214(4), 76 (2018). https://doi.org/10.1007/ s11214-018-0508-3. arXiv:1804.09294

64. Ito, Y., Ikoma, M., Kawahara, H., Nagahara, H., Kawashima, Y., Nakamoto, T.: Theoretical emission spectra of atmospheres of hot rocky super-Earths. ApJ 801(2), 144 (2015). https://doi.org/ 10.1088/0004-637X/801/2/144. arXiv:1501.05393 
65. Jin, S., Mordasini, C.: Compositional imprints in density-distance-time: a rocky composition for close-in low-mass exoplanets from the location of the valley of evaporation. ApJ 853(2), 163 (2018). https://doi.org/10.3847/1538-4357/aa9f1e. arXiv:1706.00251

66. Jontof-Hutter, D.: The compositional diversity of low-mass exoplanets. Annu. Rev. Earth Planet. Sci. 47, 141-171 (2019). https://doi.org/10.1146/annurev-earth-053018-060352. arXiv:1911.04598

67. Kaltenegger, L.: How to characterize habitable worlds and signs of life. ARA\&A 55(1), 433-485 (2017). https://doi.org/10.1146/annurev-astro-082214-122238. arXiv:1911.05597

68. Kite, E.S., Bruce, F.egley.J., Schaefer, L., Ford, E.B.: Superabundance of exoplanet sub-Neptunes explained by fugacity crisis. ApJ1 887(2), L33 (2019). https://doi.org/10.3847/2041-8213/ab59d9. arXiv: 1912.02701

69. Komacek, T.D., Youdin, A.N.: Structure and evolution of internally heated hot Jupiters. ApJ 844(2), 94 (2017). https://doi.org/10.3847/1538-4357/aa7b75. arXiv:1706.07605

70. Komacek, T.D., Thorngren, D.P., Lopez, E.D., Ginzburg, S.: Reinflation of warm and hot Jupiters. ApJ 893(1), 36 (2020). https://doi.org/10.3847/1538-4357/ab7eb4. arXiv:2003.04877

71. Kreidberg, L., Koll, D.D.B., Morley, C., Hu, R., Schaefer, L., Deming, D., Stevenson, K.B., Dittmann, J., Vanderburg, A., Berardo, D., Guo, X., Stassun, K., Crossfield, I., Charbonneau, D., Latham, D.W., Loeb, A., Ricker, G., Seager, S., Vand erspek, R.: Absence of a thick atmosphere on the terrestrial exoplanet LHS 3844b. Nature 573(7772), 87-90 (2019). https://doi.org/10.1038/ s41586-019-1497-4. arXiv:1908.06834

72. Kuchner, M.J.: Volatile-rich Earth-mass planets in the habitable zone. ApJl 596(1), L105-L108 (2003). https://doi.org/10.1086/378397. arXiv:astro-ph/0303186

73. Kunitomo, M., Guillot, T., Ida, S., Takeuchi, T.: Revisiting the pre-main-sequence evolution of stars. II. Consequences of planet formation on stellar surface composition. A\&A 618, A132 (2018). https://doi.org/10.1051/0004-6361/201833127. arXiv:1808.07396

74. Kurosaki, K., Ikoma, M., Hori, Y.: Impact of photo-evaporative mass loss on masses and radii of water-rich sub/super-Earths. A\&A 562, A80 (2014). https://doi.org/10.1051/0004-6361/201322258. arXiv:1307.3034

75. Lambrechts, M., Morbidelli, A., Jacobson, S.A., Johansen, A., Bitsch, B., Izidoro, A., Raymond, S.N.: Formation of planetary systems by pebble accretion and migration. How the radial pebble flux determines a terrestrial-planet or super-Earth growth mode. A\&A 627, A83 (2019). https://doi.org/10.1051/0004-6361/201834229. arXiv:1902.08694

76. Laughlin, G., Lissauer, J.J.: Exoplanetary geophysics—an emerging discipline. arXiv:1501.05685 (2015)

77. Laughlin, G., Crismani, M., Adams, F.C.: On the anomalous radii of the transiting extrasolar planets. ApJ1 729(1), L7 (2011). https://doi.org/10.1088/2041-8205/729/1/L7. arXiv:1101.5827

78. Leconte, J.: Continuous reorientation of synchronous terrestrial planets due to mantle convection. Nat. Geosci. 11(3), 168-172 (2018). https://doi.org/10.1038/s41561-018-0071-2. arXiv:1809.01150

79. Leconte, J., Chabrier, G.: A new vision of giant planet interiors: impact of double diffusive convection. A\&A 540, A20 (2012). https://doi.org/10.1051/0004-6361/201117595. arXiv:1201.4483

80. Ledoux, P.: Stellar models with convection and with discontinuity of the mean molecular weight. ApJ 105, 305 (1947). https://doi.org/10.1086/144905

81. Léger, A., Selsis, F., Sotin, C., Guillot, T., Despois, D., Mawet, D., Ollivier, M., Labèque, A., Valette, C., Brachet, F., Chazelas, B., Lammer, H.: A new family of planets? “ocean-planets”. Icarus 169(2), 499-504 (2004). https://doi.org/10.1016/j.icarus.2004.01.001. arXiv:astro-ph/0308324

82. Lichtenberg, T., Golabek, G.J., Gerya, T.V., Meyer, M.R.: The effects of short-lived radionuclides and porosity on the early thermo-mechanical evolution of planetesimals. Icarus 274, 350-365 (2016). https://doi.org/10.1016/j.icarus.2016.03.004. arXiv:1603.05979

83. Lichtenberg, T., Parker, R.J., Meyer, M.R.: Isotopic enrichment of forming planetary systems from supernova pollution. Mon Not R Astron Soc 462(4), 3979-3992 (2016). https://doi.org/10.1093/mnras/stw1929. arXiv:1608.01435

84. Lichtenberg, T., Golabek, G.J., Burn, R., Meyer, M.R., Alibert, Y., Gerya, T.V., Mordasini, C.: A water budget dichotomy of rocky protoplanets from ${ }^{26}$ Al-heating. Nat. Astron. 3, 307-313 (2019). https://doi.org/10.1038/s41550-018-0688-5. arXiv:1902.04026

85. Lin, D.N.C., Bodenheimer, P., Richardson, D.C.: Orbital migration of the planetary companion of 51 Pegasi to its present location. Nature 380(6575), 606-607 (1996). https://doi.org/10.1038/380606a0

86. Lopez, E.D.: Born dry in the photoevaporation desert: Kepler's ultra-short-period planets formed water-poor. MNRAS 472(1), 245-253 (2017). https://doi.org/10.1093/mnras/stx1558. arXiv: 1610.01170 
87. Lourenço, D.L., Rozel, A., Tackley, P.J.: Melting-induced crustal production helps plate tectonics on Earth-like planets. Earth Planet. Sci. Lett. 439, 18-28 (2016). https://doi.org/10.1016/ j.eps1.2016.01.024

88. Lourenço, D.L., Rozel, A.B., Gerya, T., Tackley, P.J.: Efficient cooling of rocky planets by intrusive magmatism. Nat. Geosci. 11(5), 322-327 (2018). https://doi.org/10.1038/s41561-018-0094-8

89. Lozovsky, M., Helled, R., Rosenberg, E.D., Bodenheimer, P.: Jupiter's formation and its primordial internal structure. ApJ 836, 227 (2017). https://doi.org/10.3847/1538-4357/836/2/227. arXiv:1701.01719

90. Lozovsky, M., Helled, R., Dorn, C., Venturini, J.: Threshold radii of volatile-rich planets. Astrophys. J. 866(1), 49 (2018)

91. Madhusudhan, N.: Exoplanetary atmospheres: key insights, challenges, and prospects. ARA\&A 57, 617-663 (2019). https://doi.org/10.1146/annurev-astro-081817-051846. arXiv:1904.03190

92. Madhusudhan, N., Lee, K.KM., Mousis, O.: A possible carbon-rich interior in super-Earth 55 Cancri e. ApJ1 759(2), L40 (2012). https://doi.org/10.1088/2041-8205/759/2/L40. arXiv:1210.2720

93. Madhusudhan, N., Bitsch, B., Johansen, A., Eriksson, L.: Atmospheric signatures of giant exoplanet formation by pebble accretion. MNRAS 469(4), 4102-4115 (2017). https://doi.org/10.1093/ mnras/stx1139. arXiv:1611.03083

94. Meier, T., Bower, D.J., Lichtenberg, T., Tackley, P.J.: Interior dynamics of tidally locked superEarths: the case of LHS 3844b. In: Europlanet Science Congress 2020, vol. 2020, pp. EPSC2020-778 (2020)

95. Miguel, Y.: Observability of molecular species in a nitrogen dominated atmosphere for 55 Cancri e. MNRAS 482(3), 2893-2901 (2019). https://doi.org/10.1093/mnras/sty2803. arXiv:1809.08230

96. Miguel, Y., Kaltenegger, L., Fegley, B., Schaefer, L.: Compositions of hot super-Earth atmospheres: exploring Kepler candidates. ApJ1 742(2), L19 (2011). https://doi.org/10.1088/2041-8205/ 742/2/L19. arXiv:1110.2426

97. Miller, N., Fortney, J.J.: The Heavy-element masses of extrasolar giant planets, revealed. ApJl 736(2), L29 (2011). https://doi.org/10.1088/2041-8205/736/2/L29. arXiv:1105.0024

98. Miozzi, F., Morard, G., Antonangeli, D., Clark, A.N., Mezouar, M., Dorn, C., Rozel, A., Fiquet, G.: Equation of state of $\mathrm{SiC}$ at extreme conditions: new insight into the interior of carbon-rich exoplanets. J. Geophys. Res. (Planets) 123(9), 2295-2309 (2018). https://doi.org/10.1029/2018JE005582. arXiv: 1808.08201

99. Miyagoshi, T., Kameyama, M., Ogawa, M.: Effects of adiabatic compression on thermal convection in super-Earths of various sizes. Earth Planets Space 70(1), 200 (2018). https://doi.org/10.1186/ s40623-018-0975-5

100. Mizuno, H.: Formation of the giant planets. Prog. Theor. Phys. 64(2), 544-557 (1980). https:// doi.org/10.1143/PTP.64.544

101. Mol Lous, M., Miguel, Y.: Inflation of migrated hot Jupiters. MNRAS 495(3), 2994-3001 (2020). https://doi.org/10.1093/mnras/staa1405. arXiv:2005.04927

102. Morales, M.A., Schwegler, E., Ceperley, D., Pierleoni, C., Hamel, S., Caspersen, K.: Phase separation in hydrogen-helium mixtures at Mbar pressures. Proc. Natl. Acad. Sci. 106, 1324 (2009). https://doi.org/10.1073/pnas.0812581106. arXiv:0903.0980

103. Müller, S., Ben-Yami, M., Helled, R.: Theoretical versus observational uncertainties: composition of giant exoplanets. ApJ 903(2), 147 (2020). https://doi.org/10.3847/1538-4357/abba19. arXiv:2009.09746

104. Müller, S., Helled, R., Cumming, A.: The challenge of forming a fuzzy core in Jupiter. arXiv:2004.13534 (2020)

105. Ni, D.: Understanding Jupiter's deep interior: the effect of a dilute core. A\&A 632, A76 (2019). https://doi.org/10.1051/0004-6361/201935938

106. Öberg, K.I., Bergin, E.A.: Excess c/o and c/h in outer protoplanetary disk gas. Astrophys. J. Lett. 831(2), L19 (2016)

107. Öberg, K.I., Wordsworth, R.: Jupiter's composition suggests its core assembled exterior to the $\mathrm{N}_{2}$ snowline. AJ 158(5), 194 (2019). https://doi.org/10.3847/1538-3881/ab46a8. arXiv:1909.11246

108. Öberg, K.I., Murray-Clay, R., Bergin, E.A.: The effects of snowlines on C/O in planetary atmospheres. ApJ1 743(1), L16 (2011). https://doi.org/10.1088/2041-8205/743/1/L16. arXiv:1110.5567

109. Olson, P.L., Sharp, Z.D.: Nebular atmosphere to magma ocean: a model for volatile capture during Earth accretion. Phys. Earth Planet. Inter. 294, 106294 (2019). https://doi.org/10.1016/ j.pepi.2019.106294 
110. O’Neill, C., Lenardic, A., Weller, M., Moresi, L., Quenette, S., Zhang, S.: A window for plate tectonics in terrestrial planet evolution? Phys. Earth Planet. Inter. 255, 80-92 (2016). https://doi.org/10.1016/j.pepi.2016.04.002

111. Otegi, J.F., Bouchy, F., Helled, R.: Revisited mass-radius relations for exoplanets below $120 \mathrm{M}_{\oplus}$. A\&A 634, A43 (2020). https://doi.org/10.1051/0004-6361/201936482. arXiv:1911.04745

112. Owen, J.E., Wu, Y.: The evaporation valley in the Kepler planets. ApJ 847(1), 29 (2017). https://doi.org/10.3847/1538-4357/aa890a. arXiv:1705.10810

113. Parmentier, V., Fortney, J.J., Showman, A.P., Morley, C., Marley, M.S.: Transitions in the cloud composition of hot Jupiters. ApJ 828(1), 22 (2016). https://doi.org/10.3847/0004-637X/828/1/22. arXiv: 1602.03088

114. Perna, R., Menou, K., Rauscher, E.: Ohmic dissipation in the atmospheres of hot Jupiters. ApJ 724(1), 313-317 (2010). https://doi.org/10.1088/0004-637X/724/1/313. arXiv:1009.3273

115. Pinhas, A., Madhusudhan, N., Gandhi, S., MacDonald, R.: $\mathrm{H}_{2} \mathrm{O}$ abundances and cloud properties in ten hot giant exoplanets. MNRAS 482(2), 1485-1498 (2019). https://doi.org/10.1093/mnras/sty2544. arXiv:1811.00011

116. Plotnykov, M., Valencia, D.: Chemical fingerprints of formation in rocky super-earths' data. Mon. Not. R. Astron. Soc. 499(1), 932-947 (2020)

117. Rauer, H., Catala, C., Aerts, C., Appourchaux, T., Benz, W., Brandeker, A., Christensen-Dalsgaard, J., Deleuil, M., Gizon, L., Goupil, M.J., Güdel, M., Janot-Pacheco, E., Mas-Hesse, M., Pagano, I., Piotto, G., Pollacco, D., Santos, C., Smith, A., Suárez, J.C., Szabó, R., Udry, S., Adibekyan, V., Alibert, Y., Almenara, J.M., Amaro-Seoane, P., Eiff, M.AV., Asplund, M., Antonello, E., Barnes, S., Baudin, F., Belkacem, K., Bergemann, M., Bihain, G., Birch, A.C., Bonfils, X., Boisse, I., Bonomo, A.S., Borsa, F., Brandão, I.M., Brocato, E., Brun, S., Burleigh, M., Burston, R., Cabrera, J., Cassisi, S., Chaplin, W., Charpinet, S., Chiappini, C., Church, R.P., Csizmadia, S., Cunha, M., Damasso, M., Davies, M.B., Deeg, H.J., Díaz, R.F., Dreizler, S., Dreyer, C., Eggenberger, P., Ehrenreich, D., Eigmüller, P., Erikson, A., Farmer, R., Feltzing, S., de Oliveira Fialho, F., Figueira, P., Forveille, T., Fridlund, M., García, R.A., Giommi, P., Giuffrida, G., Godolt, M., Gomes da Silva, J., Granzer, T., Grenfell, J.L., Grotsch-Noels, A., Günther, E., Haswell, C.A., Hatzes, A.P., Hébrard, G., Hekker, S., Helled, R., Heng, K., Jenkins, J.M., Johansen, A., Khodachenko, M.L., Kislyakova, K.G., Kley, W., Kolb, U., Krivova, N., Kupka, F., Lammer, H., Lanza, A.F., Lebreton, Y., Magrin, D., Marcos-Arenal, P., Marrese, P.M., Marques, J.P., Martins, J., Mathis, S., Mathur, S., Messina, S., Miglio, A., Montalban, J., Montalto, M., Monteiro, M.JPFG., Moradi, H., Moravveji, E., Mordasini, C., Morel, T., Mortier, A., Nascimbeni, V., Nelson, R.P., Nielsen, M.B., Noack, L., Norton, A.J., Ofir, A., Oshagh, M., Ouazzani, R.M., Pápics, P., Parro, V.C., Petit, P., Plez, B., Poretti, E., Quirrenbach, A., Ragazzoni, R., Raimondo, G., Rainer, M., Reese, D.R., Redmer, R., Reffert, S., Rojas-Ayala, B., Roxburgh, I.W., Salmon, S., Santerne, A., Schneider, J., Schou, J., Schuh, S., Schunker, H., Silva-Valio, A., Silvotti, R., Skillen, I., Snellen, I., Sohl, F., Sousa, S.G., Sozzetti, A., Stello, D., Strassmeier, K.G., Švanda, M., Szabó, G.M., Tkachenko, A., Valencia, D., Van Grootel, V., Vauclair, S.D., Ventura, P., Wagner, F.W., Walton, N.A., Weingrill, J., Werner, S.C., Wheatley, P.J., Zwintz, K.: The PLATO 2.0 mission. Exp. Astron. 38(1-2), 249-330 (2014). https://doi.org/10.1007/s10686-014-9383-4. arXiv: 1310.0696

118. Rauscher, E., Menou, K.: Three-DIMENSIONAL ATMOSPHERIC CIRCULATION Models of HD 189733b and HD 209458b with consistent magnetic drag and ohmic dissipation. ApJ 764(1), 103 (2013). https://doi.org/10.1088/0004-637X/764/1/103. arXiv:1208.2274

119. Rauscher, E., Showman, A.P.: The influence of differential irradiation and circulation on the thermal evolution of gas giant planets. I. Upper limits from radiative equilibrium. ApJ 784(2), 160 (2014). https://doi.org/10.1088/0004-637X/784/2/160. arXiv:1309.7052

120. Rimmer, P.B., Rugheimer, S.: Hydrogen cyanide in nitrogen-rich atmospheres of rocky exoplanets. Icarus 329, 124-131 (2019)

121. Rogers, T.M., Showman, A.P.: Magnetohydrodynamic simulations of the atmosphere of HD 209458b. ApJ1 782(1), L4 (2014). https://doi.org/10.1088/2041-8205/782/1/L4. arXiv:1401.5815

122. Rubie, D.C., Jacobson, S.A., Morbidelli, A., O’Brien, D.P., Young, E.D., de Vries, J., Nimmo, F., Palme, H., Frost, D.J.: Accretion and differentiation of the terrestrial planets with implications for the compositions of early-formed Solar System bodies and accretion of water. Icarus 248, 89-108 (2015). https://doi.org/10.1016/j.icarus.2014.10.015. arXiv:1410.3509

123. Sainsbury-Martinez, F., Wang, P., Fromang, S., Tremblin, P., Dubos, T., Meurdesoif, Y., Spiga, A., Leconte, J., Baraffe, I., Chabrier, G., Mayne, N., Drummond, B., Debras, F.: Idealised simulations 
of the deep atmosphere of hot Jupiters. Deep, hot adiabats as a robust solution to the radius inflation problem. A\&A 632, A114 (2019). https://doi.org/10.1051/0004-6361/201936445. arXiv:1911.06546

124. Salvador, A., Massol, H., Davaille, A., Marcq, E., Sarda, P., Chassefière, E.: The relative influence of $\mathrm{H}_{2} \mathrm{O}$ and $\mathrm{CO}_{2}$ on the primitive surface conditions and evolution of rocky planets. J. Geophys. Res. (Planets) 122(7), 1458-1486 (2017). https://doi.org/10.1002/2017JE005286

125. Sato, B., Fischer, D.A., Henry, G.W., Laughlin, G., Butler, R.P., Marcy, G.W., Vogt, S.S., Bodenheimer, P., Ida, S., Toyota, E., Wolf, A., Valenti, J.A., Boyd, L.J., Johnson, J.A., Wright, J.T., Ammons, M., Robinson, S., Strader, J., McCarthy, C., Tah, K.L., Minniti, D.: The N2K Consortium. II. A transiting hot Saturn around HD 149026 with a large dense core. ApJ 633(1), 465-473 (2005). https://doi.org/10.1086/449306. arXiv:astro-ph/0507009

126. Schaefer, L., Bruce, F.J.: Redox states of initial atmospheres outgassed on rocky planets and planetesimals. ApJ 843(2), 120 (2017). https://doi.org/10.3847/1538-4357/aa784f

127. Schaefer, L., Elkins-Tanton, L.T.: Magma oceans as a critical stage in the tectonic development of rocky planets. Philos. Trans. R. Soc. Lond. Ser. A 376(2132), 20180109 (2018). https://doi.org/10.1098/rsta.2018.0109. arXiv:1809.01629

128. Schaefer, L., Fegley, B.: Chemistry of silicate atmospheres of evaporating super-Earths. ApJ1 703(2), L113-L117 (2009). https://doi.org/10.1088/0004-637X/703/2/L113. arXiv:0906.1204

129. Schaefer, L., Lodders, K., Fegley, B.: Vaporization of the Earth: application to exoplanet atmospheres. ApJ 755(1), 41 (2012). https://doi.org/10.1088/0004-637X/755/1/41. arXiv:1108.4660

130. Schaefer, L., Wordsworth, R.D., Berta-Thompson, Z., Sasselov, D.: Predictions of the Atmospheric Composition of GJ. ApJ 829(2), 63 (1132b). https://doi.org/10.3847/0004-637X/829/2/63. arXiv: 1607.03906

131. Schulze, J., Wang, J., Johnson, J., Unterborn, C., Panero, W.: The probability that a rocky planet's composition reflects its host star. arXiv:201108893 (2020)

132. Shibata, S., Ikoma, M.: Capture of solids by growing proto-gas giants: effects of gap formation and supply limited growth. MNRAS 487(4), 4510-4524 (2019). https://doi.org/10.1093/mnras/stz1629. arXiv: 1906.05530

133. Shibata, S., Helled, R., Ikoma, M.: The origin of the high metallicity of close-in giant exoplanets. Combined effects of resonant and aerodynamic shepherding. A\&A 633, A33 (2020). https://doi.org/10.1051/0004-6361/201936700. arXiv:1911.02292

134. Showman, A.P., Guillot, T.: Atmospheric circulation and tides of " 51 Pegasus b-like" planets. A\&A 385, 166-180 (2002). https://doi.org/10.1051/0004-6361:20020101

135. Soubiran, F., Militzer, B., Driver, K.P., Zhang, S.: Properties of hydrogen, helium, and silicon dioxide mixtures in giant planet interiors. Phys. Plasmas 24(4), 041401 (2017). https://doi.org/10.1063/ 1.4978618. arXiv: 1703.09840

136. Spaargaren, R.J., Ballmer, M.D., Bower, D.J., Dorn, C., Tackley, P.J.: The influence of bulk composition on the long-term interior-atmosphere evolution of terrestrial exoplanets. Astron. Astrophys. 643, A44 (2020)

137. Spiegel, D.S., Fortney, J.J., Sotin, C.: Structure of exoplanets. Proc. Natl. Acad. Sci. 111(35), 1262212627 (2014). https://doi.org/10.1073/pnas.1304206111. arXiv:1312.3323

138. Stevenson, D.J., Salpeter, E.E.: The dynamics and helium distribution in hydrogen-helium fluid planets. ApJs 35, 239-261 (1977). https://doi.org/10.1086/190479

139. Tackley, P.J., Ammann, M., Brodholt, J.P., Dobson, D.P., Valencia, D.: Mantle dynamics in superEarths: post-perovskite rheology and self-regulation of viscosity. Icarus 225(1), 50-61 (2013). https://doi.org/10.1016/j.icarus.2013.03.013. arXiv:1204.3539

140. Tanaka, H., Takeuchi, T., Ward, W.R.: Three-dimensional interaction between a planet and an isothermal gaseous disk. I. Corotation and Lindblad Torques and planet migration. ApJ 565(2), 1257-1274 (2002). https://doi.org/10.1086/324713

141. Thorngren, D.P., Fortney, J.J.: Bayesian analysis of hot-Jupiter radius anomalies: evidence for ohmic dissipation? AJ 155(5), 214 (2018). https://doi.org/10.3847/1538-3881/aaba13. arXiv:1709.04539

142. Thorngren, D.P., Fortney, J.J., Murray-Clay, R.A., Lopez, E.D.: The mass-metallicity relation for giant planets. ApJ 831(1), 64 (2016). https://doi.org/10.3847/0004-637X/831/1/64. arXiv: 1511.07854

143. Tinetti, G., Drossart, P., Eccleston, P., Hartogh, P., Heske, A., Leconte, J., Micela, G., Ollivier, M., Pilbratt, G., Puig, L., Turrini, D., Vandenbussche, B., Wolkenberg, P., Beaulieu, J.P., Buchave, L.A., Ferus, M., Griffin, M., Guedel, M., Justtanont, K., Lagage, P.O., Machado, P., Malaguti, G., Min, M., Nørgaard-Nielsen, H.U., Rataj, M., Ray, T., Ribas, I., Swain, M., Szabo, R., Werner, S., Barstow, J., 
Burleigh, M., Cho, J., du Foresto, V.C., Coustenis, A., Decin, L., Encrenaz, T., Galand, M., Gillon, M., Helled, R., Morales, J.C., Muñoz, A.G., Moneti, A., Pagano, I., Pascale, E., Piccioni, G., Pinfield, D., Sarkar, S., Selsis, F., Tennyson, J., Triaud, A., Venot, O., Waldmann, I., Waltham, D., Wright, G., Amiaux, J., Auguères, J.L., Berthé, M., Bezawada, N., Bishop, G., Bowles, N., Coffey, D., Colomé, J., Crook, M., Crouzet, P.E., Da Peppo, V., Sanz, I.E., Focardi, M., Frericks, M., Hunt, T., Kohley, R., Middleton, K., Morgante, G., Ottensamer, R., Pace, E., Pearson, C., Stamper, R., Symonds, K., Rengel, M., Renotte, E., Ade, P., Affer, L., Alard, C., Allard, N., Altieri, F., André, Y., Arena, C., Argyriou, I., Aylward, A., Baccani, C., Bakos, G., Banaszkiewicz, M., Barlow, M., Batista, V., Bellucci, G., Benatti, S., Bernardi, P., Bézard, B., Blecka, M., Bolmont, E., Bonfond, B., Bonito, R., Bonomo, A.S., Brucato, J.R., Brun, A.S., Bryson, I., Bujwan, W., Casewell, S., Charnay, B., Pestellini, C.C., Chen, G., Ciaravella, A., Claudi, R., Clédassou, R., Damasso, M., Damiano, M., Danielski, C., Deroo, P., Di Giorgio, A.M., Dominik, C., Doublier, V., Doyle, S., Doyon, R., Drummond, B., Duong, B., Eales, S., Edwards, B., Farina, M., Flaccomio, E., Fletcher, L., Forget, F., Fossey, S., Fränz, M., Fujii, Y., García-Piquer, Á., Gear, W., Geoffray, H., Gérard, J.C., Gesa, L., Gomez, H., Graczyk, R., Griffith, C., Grodent, D., Guarcello, M.G., Gustin, J., Hamano, K., Hargrave, P., Hello, Y., Heng, K., Herrero, E., Hornstrup, A., Hubert, B., Ida, S., Ikoma, M., Iro, N., Irwin, P., Jarchow, C., Jaubert, J., Jones, H., Julien, Q., Kameda, S., Kerschbaum, F., Kervella, P., Koskinen, T., Krijger, M., Krupp, N., Lafarga, M., Landini, F., Lellouch, E., Leto, G., Luntzer, A., Rank-Lüftinger, T., Maggio, A., Maldonado, J., Maillard, J.P., Mall, U., Marquette, J.B., Mathis, S., Maxted, P., Matsuo, T., Medvedev, A., Miguel, Y., Minier, V., Morello, G., Mura, A., Narita, N., Nascimbeni, V., Nguyen Tong, N., Noce, V., Oliva, F., Palle, E., Palmer, P., Pancrazzi, M., Papageorgiou, A., Parmentier, V., Perger, M., Petralia, A., Pezzuto, S., Pierrehumbert, R., Pillitteri, I., Piotto, G., Pisano, G., Prisinzano, L., Radioti, A., Réess, J.M., Rezac, L., Rocchetto, M., Rosich, A., Sanna, N., Santerne, A., Savini, G., Scandariato, G., Sicardy, B., Sierra, C., Sindoni, G., Skup, K., Snellen, I., Sobiecki, M., Soret, L., Sozzetti, A., Stiepen, A., Strugarek, A., Taylor, J., Taylor, W., Terenzi, L., Tessenyi, M., Tsiaras, A., Tucker, C., Valencia, D., Vasisht, G., Vazan, A., Vilardell, F., Vinatier, S., Viti, S., Waters, R., Wawer, P., Wawrzaszek, A., Whitworth, A., Yung, Y.L., Yurchenko, S.N., Osorio, M.RZ., Zellem, R., Zingales, T., Zwart, F.: A chemical survey of exoplanets with ARIEL. Exp. Astron. 46(1), 135-209 (2018). https://doi.org/10.1007/s10686-018-9598-X

144. Tremblin, P., Chabrier, G., Mayne, N.J., Amundsen, D.S., Baraffe, I., Debras, F., Drummond, B., Manners, J., Fromang, S.: Advection of potential temperature in the atmosphere of irradiated exoplanets: a robust mechanism to explain radius inflation. ApJ 841(1), 30 (2017). https://doi.org/10.3847/1538-4357/aa6e57. arXiv:1704.05440

145. Trønnes, R.G., Baron, M.A., Eigenmann, K.R., Guren, M.G., Heyn, B.H., Løken, A., Mohn, C.E.: Core formation, mantle differentiation and core-mantle interaction within Earth and the terrestrial planets. Tectonophysics 760, 165-198 (2019). https://doi.org/10.1016/j.tecto.2018.10.021

146. Tsiaras, A., Rocchetto, M., Waldmann, I.P., Venot, O., Varley, R., Morello, G., Damiano, M., Tinetti, G., Barton, E.J., Yurchenko, S.N., Tennyson, J.: Detection of an atmosphere around the super-Earth 55 Cancri e. ApJ 820(2), 99 (2016). https://doi.org/10.3847/0004-637X/820/2/99. arXiv:1511.08901

147. Tsiganis, K., Gomes, R., Morbidelli, A., Levison, H.F.: Origin of the orbital architecture of the giant planets of the Solar System. Nature 435(7041), 459-461 (2005). https://doi.org/10.1038/nature03539

148. Turrini, D., Miguel, Y., Zingales, T., Piccialli, A., Helled, R., Vazan, A., Oliva, F., Sindoni, G., Panić, O., Leconte, J., Min, M., Pirani, S., Selsis, F., Coudé du Foresto, V., Mura, A., Wolkenberg, P.: The contribution of the ARIEL space mission to the study of planetary formation. Exp. Astron. 46(1), 45-65 (2018). https://doi.org/10.1007/s10686-017-9570-1. arXiv:1804.06179

149. Unterborn, C.T., Panero, W.R.: The effects of $\mathrm{mg} / \mathrm{si}$ on the exoplanetary refractory oxygen budget. Astrophys. J. 845(1), 61 (2017)

150. Unterborn, C.T., Hull, S.D., Stixrude, L.P., Teske, J.K., Johnson, J.A., Panero, W.R.: Stellar chemical clues as to the rarity of exoplanetary tectonics. arXiv:170610282 (2017)

151. Valencia, D., Sasselov, D.D., O’Connell, R.J.: Radius and structure models of the first super-Earth planet. ApJ 656(1), 545-551 (2007). https://doi.org/10.1086/509800. arXiv:astro-ph/0610122

152. Valencia, D., Guillot, T., Parmentier, V., Freedman, R.S.: Bulk composition of GJ $1214 \mathrm{~b}$ and other sub-Neptune exoplanets. ApJ 775(1), 10 (2013). https://doi.org/10.1088/0004-637X/775/1/10. arXiv: 1305.2629

153. Valletta, C., Helled, R.: The distribution of heavy-elements in giant protoplanetary atmospheres: the importance of planetesimal-envelope interactions. arXiv:1811.10904 (2018) 
154. Valletta, C., Helled, R.: Giant planet formation models with a self-consistent treatment of the heavy elements. ApJ 900(2), 133 (2020). https://doi.org/10.3847/1538-4357/aba904. arXiv:2007.13577

155. van Summeren, J., Conrad, C.P., Gaidos, E.: Mantle convection, plate tectonics, and volcanism on hot exo-Earths. ApJ1 736(1), L15 (2011). https://doi.org/10.1088/2041-8205/736/1/L15. arXiv: 1106.4341

156. Vazan, A., Kovetz, A., Podolak, M., Helled, R.: The effect of composition on the evolution of giant and intermediate-mass planets. MNRAS 434, 3283-3292 (2013). https://doi.org/ 10.1093/mnras/stt1248. arXiv:1307.2033

157. Vazan, A., Helled, R., Kovetz, A., Podolak, M.: Convection and mixing in giant planet evolution. ApJ 803, 32 (2015). https://doi.org/10.1088/0004-637X/803/1/32. arXiv:1502.03270

158. Vazan, A., Helled, R., Podolak, M., Kovetz, A.: The evolution and internal structure of Jupiter and Saturn with compositional gradients. ApJ 829, 118 (2016). https://doi.org/10.3847/0004637X/829/2/118. arXiv:1606.01558

159. Vazan, A., Helled, R., Guillot, T.: Jupiter's evolution with primordial composition gradients. A\&A 610, L14 (2018). https://doi.org/10.1051/0004-6361/201732522. arXiv:1801.08149

160. Vazan, A., Ormel, C.W., Noack, L., Dominik, C.: Contribution of the core to the thermal evolution of sub-Neptunes. ApJ 869(2), 163 (2018). https://doi.org/10.3847/1538-4357/aaef33. arXiv: 1811.02588

161. Venturini, J., Helled, R.: The formation of mini-Neptunes. ApJ 848(2), 95 (2017). https://doi.org/10.3847/1538-4357/aa8cd0. arXiv:1709.04736

162. Wahl, S.M., Wilson, H.F., Militzer, B.: Solubility of iron in metallic hydrogen and stability of dense cores in giant planets. ApJ 773, 95 (2013). https://doi.org/10.1088/0004-637X/773/2/95. arXiv: 1303.6743

163. Wahl, S.M., Hubbard, W.B., Militzer, B., Guillot, T., Miguel, Y., Movshovitz, N., Kaspi, Y., Helled, R., Reese, D., Galanti, E., Levin, S., Connerney, J.E., Bolton, S.J.: Comparing Jupiter interior structure models to Juno gravity measurements and the role of a dilute core. Geophys. Res. Lett. 44(10), 4649-4659 (2017). https://doi.org/10.1002/2017GL073160. arXiv:1707.01997

164. Wang, H.S., Lineweaver, C.H., Ireland, T.R.: The volatility trend of protosolar and terrestrial elemental abundances. Icarus 328, 287-305 (2019)

165. Williams, C.D., Mukhopadhyay, S.: Capture of nebular gases during Earth's accretion is preserved in deep-mantle neon. Nature 565(7737), 78-81 (2019). https://doi.org/10.1038/s41586-018-0771-1

166. Wilson, H.F., Militzer, B.: Solubility of water ice in metallic hydrogen: consequences for core erosion in gas giant planets. ApJ 745, 54 (2012). https://doi.org/10.1088/0004-637X/745/1/54

167. Wordsworth, R., Pierrehumbert, R.: Hydrogen-nitrogen greenhouse warming in earth's early atmosphere. Science 339(6115), 64-67 (2013)

168. Wu, Y., Lithwick, Y.: Ohmic heating suspends, not reverses, the cooling contraction of hot Jupiters. ApJ 763(1), 13 (2013). https://doi.org/10.1088/0004-637X/763/1/13. arXiv:1202.0026

169. Youdin, A.N., Mitchell, J.L.: The mechanical greenhouse: burial of heat by turbulence in hot Jupiter atmospheres. ApJ 721(2), 1113-1126 (2010). https://doi.org/10.1088/0004-637X/721/2/1113. arXiv: 1008.0645

170. Zeng, L., Jacobsen, S.B., Sasselov, D.D., Petaev, M.I., Vanderburg, A., Lopez-Morales, M., PerezMercader, J., Mattsson, T.R., Li, G., Heising, M.Z., Bonomo, A.S., Damasso, M., Berger, T.A., Cao, H., Levi, A., Wordsworth, R.D.: Growth model interpretation of planet size distribution. Proc. Natl. Acad. Sci. 116(20), 9723-9728 (2019). https://doi.org/10.1073/pnas.1812905116. arXiv:1906.04253

171. Zilinskas, M., Miguel, Y., Mollière, P., Tsai, S.M.: Atmospheric compositions and observability of nitrogen-dominated ultra-short-period super-Earths. MNRAS 494(1), 1490-1506 (2020). https://doi.org/10.1093/mnras/staa724. arXiv:2003.05354

Publisher's note Springer Nature remains neutral with regard to jurisdictional claims in published maps and institutional affiliations. 


\section{Affiliations}

Ravit Helled ${ }^{1}$ (D) . Stephanie Werner ${ }^{2} \cdot$ Caroline Dorn $^{1}$ (D) $\cdot$ Tristan Guillot $^{3}$ Masahiro Ikoma ${ }^{4}$ (D) . Yuichi Ito ${ }^{5}$ (D) . Mihkel Kama ${ }^{6}$. Tim Lichtenberg ${ }^{7}$ (D) Yamila Miguel $^{8,9}$ (1) . Oliver Shorttle ${ }^{10}$. Paul J. Tackley ${ }^{11}$. Diana Valencia ${ }^{12}$ (i) Allona Vazan ${ }^{13}$

1 Institute for Computational Science, University of Zurich, Winterthurerstr. 190, CH-8057 Zurich, Switzerland

2 Department of Geosciences, University of Oslo, PO 1028 Blindern, 0315 Oslo, Norway

3 Observatoire de la Côte d'Azur/CNRS, 6304 Nice Cedex 4, France

4 Department of Earth and Planetary Science, The University of Tokyo, Tokyo 113-0033, Japan

5 Department of Physics and Astronomy, University College London, London, WC1E 6BT, UK

6 Tartu Observatory, University of Tartu, Observatooriumi 1, 61602, Tõravere, Estonia

7 Atmospheric, Oceanic and Planetary Physics, Department of Physics, University of Oxford, Oxford, UK

8 Leiden Observatory, University of Leiden, Niels Bohrweg 2, 2333CA Leiden, The Netherlands

9 SRON Netherlands Institute for Space Research, Sorbonnelaan 2, NL-3584 CA Utrecht, The Netherlands

10 Institute of Astronomy, University of Cambridge, Cambridge, UK

11 Institute of Geophysics, ETH Zurich, 8092, Zurich, Switzerland

12 University of Toronto, Toronto, Canada

13 Astrophysical Research Center of the Open university (ARCO), Department of Natural Sciences, The Open University of Israel, Ra' anana, Israel 